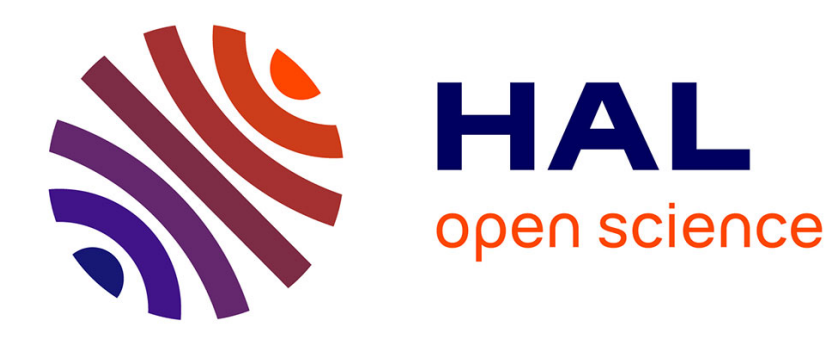

\title{
Rescaling of the Roe Scheme in Low Mach-Number Flow Regions \\ Jean-Christophe Boniface
}

\section{To cite this version:}

Jean-Christophe Boniface. Rescaling of the Roe Scheme in Low Mach-Number Flow Regions. 2016. hal-01286182v2

\author{
HAL Id: hal-01286182 \\ https://hal.science/hal-01286182v2
}

Preprint submitted on 1 Apr 2016

HAL is a multi-disciplinary open access archive for the deposit and dissemination of scientific research documents, whether they are published or not. The documents may come from teaching and research institutions in France or abroad, or from public or private research centers.
L'archive ouverte pluridisciplinaire HAL, est destinée au dépôt et à la diffusion de documents scientifiques de niveau recherche, publiés ou non, émanant des établissements d'enseignement et de recherche français ou étrangers, des laboratoires publics ou privés. 


\title{
Rescaling of the Roe Scheme in Low Mach-Number Flow Regions
}

\author{
Jean-Christophe Boniface
}

ONERA - The French Aerospace Lab, F-92190, Meudon, France

\begin{abstract}
A rescaled matrix-valued dissipation is reformulated for the Roe scheme in low Mach-number flow regions from a well known family of local low-speed preconditioners popularized by Turkel. The rescaling is obtained by suppressing the iterative preconditioning and by deriving explicitly the full set of eigenspaces of the Roe-Turkel matrix dissipation. This formulation preserves the time consistency and does not require to reformulate the boundary conditions based on the characteristic theory. The dissipation matrix achieves by construction the proper scaling in low-speed flow regions and returns the original Roe scheme at the sonic line. We find that all eigenvalues are nonnegative in the subsonic regime. By removing the iterative preconditioning, it becomes necessary to formulate a stringent stability condition to the explicit scheme in the low-speed flow regions based on the spectral radius of the rescaled matrix dissipation. This formulation also raises a two-timescale problem for the acoustic waves, which is circumvented for a steady-state iterative procedure by the development of a robust implicit characteristic matrix time-stepping scheme. The behaviour of the modified eigenvalues in the incompressible limit and at the sonic line also suggest applying the entropy correction carefully for complex non-linear flows.
\end{abstract}

Keywords: Compressible flow; Euler equations; Low Mach number; Preconditioning; Roe scheme; Roe-Turkel scheme; Characteristic time-step

\section{Introduction}

Iterative preconditioning based on Chorin artificial compressibility has become widely used for computing low-speed flow configurations with numerical schemes developed for compressible flows. This iterative preconditioning technique was designed to achieve an optimal conditioning of the iterative procedure and to guaranty the proper scaling of the artificial dissipation

Email address: jean-christophe.bonif ace@onera.fr () 
when the Mach number approaches zero. The low-speed preconditioning approach has proved to be very efficient to overcome the accuracy issue of compressible flow solvers in the incompressible limit. Actually, the low-speed preconditioning should always be used since many industrial applications are characterized by mixed compressible and incompressible flows, over a wide range of Reynolds numbers.

However, the standard iterative preconditioning suffers from the complexity of its practical implementation. Since the local preconditioner modifies the characteristic relations, all boundary conditions based on characteristic variables or Riemann invariants must be reformulated accordingly. For large aerodynamics codes in which a large number of boundary conditions may be implemented, it is then necessary to reformulate most of the boundary conditions. Furthermore, the extension of iterative preconditioning to unsteady flows is not trivial and without a special treatment, time-accuracy may be lost.

The problem of the accuracy in the asymptotic limit of the incompressible flow for the discretization of the normalized Euler equations was first addressed by Guillard and Viozat [1,2]. For their non-iterative preconditioning formulated with the Roe scheme as defined in [2], it was shown that the checkerboard modes for the leading and second-order pressure fields are cancelled out by the rescaled matrix dissipation and that the pressure field should be constant in space up to a fluctuation in space of order two. Furthermore, the authors have clearly pointed out a lack of dissipation of the standard conservative schemes in the incompressible limit.

A number of authors have further considered the discrete analysis based on the normalized equations for the asymptotic behaviour of the pressure and velocity fields. An "all-speed Roe scheme" has been developed by $\mathrm{Li}$ and $\mathrm{Gu}$ [3] in order to recover at the discrete level the divergence constraint of the leading order velocity and the Poisson equation for the second-order pressure, which are not satisfied by the rescaling of the Roe scheme formulated in $[1,2]$. However, checkerboards modes are not automatically suppressed by their numerical flux and a low Mach number fix proposed by Rieper for the Roe's approximate Riemann solver [4] seems attractive, as combining the advantages in the incompressible limit of both approaches investigated in [1] and [3].

Over the last years, many modified Roe-type [1], AUSM-type [5] or Godunov-type schemes [6] have been formulated to apply conservative finite-difference schemes to low-speed flows. It has been found necessary to propose a unified theoretical framework to analyse their respective 
discrete properties and to understand why they fail to be accurate in the incompressible limit without specific corrections. It is worth mentioning the work of $\mathrm{Li}$ and $\mathrm{Gu}$ for the analysis of Roe-type schemes based on expansions in the freestream mach number of solutions of the normalized Euler equations [7] and the contribution of Dellacherie for Godunov-type schemes [8] using the Hodge decomposition for solutions derived from the one-dimensional barotropic Euler equations.

On the other hand, few contributions have addressed the numerical stability of shock-capturing schemes adapted for low-speed flows. A Fourier Analysis is carried out by Dellacherie for the one-dimensional wave equations using the low Mach Godunov scheme and an explicit CFL condition is formulated for both the explicit and the implicit scheme [8]. For a non-iterative lowspeed preconditioning formulation of the compressible Euler equations, the issue of the stability for a matrix-valued dissipation formulated from the Lax-Friedrich scheme is pointed out for the first time by Birken and Meister [9] on the basis of the asymptotic behaviour of the largest eigenvalue in the incompressible limit. Formulating a stability criteria is also an essential feature when designing numerical schemes for complex flows, especially when an "all-speed scheme" is being developed. Results obtained by Birken and Meister clearly show for the Euler equations that the standard CFL condition used for the computation of compressible flows is no longer valid in the incompressible limit and that a stringent stability condition for the time step with $\Delta t \simeq O\left(M^{2}\right)$ when $M \rightarrow 0$ must be accounted for when a non-iterative preconditioning is considered. Nevertheless, the eigenspaces of the matrix-valued dissipation are not derived and a practical CFL condition for the local time step is not formulated explicitly for the steady-state problem.

Thus, a practical point of view has essentially motivated this contribution. For the compressible Euler equations, it was found interesting to investigate the effect of removing the premultiplication of the preconditioning matrix with the time-derivative of the independent flow variables. This formulation called "non-iterative preconditioning" and corresponding to a rescaling of the matrix-valued dissipation has been investigated particularly in [1, 2] and [9]. With this simplification, the explicit scheme recovers a basic structure with the centred scheme and stabilization terms. Then it becomes no longer necessary to reformulate the characteristic curves for the preconditioned Jacobian matrix and the time accuracy is preserved. Some recent attempts of improving the accuracy of conservative schemes in the low speed limit as mentioned above may have been also motivated by a drastic simplification of the implementation of the low-speed 
preconditioning. This is especially the case of the Rieper low Mach-number fix proposed in [4], and the Thornber et al. "Low Mach" LMRoe scheme [10] modifying jumps of the discrete velocities, which was further developed by Oßwald et al. with the "Low dissipation Low Mach" $L^{2}$ Roe scheme [11]. This may also explain the success of the AUSM-familly schemes and their modification for low Mach-number flows.

The stabilization terms must be then interpreted as a rescaling of the original dissipation in the low-speed flow regions and therefore the necessary Von Neumann criteria for the linear stability must be reconsidered completely. This can be achieved only by deriving the eigenvalues and the full set of the right and left eigenvectors of the matrix-valued dissipation. The diagonalization is performed step by step, by considering first as working variables the symmetrising entropic variables for the stream-aligned formulation of the Euler equations. The formulation for the conservative variables is obtained by successive similarity transformations. We have considered the Roe scheme as baseline formulation for the matrix dissipation. The necessary rescaling of the Roe scheme in the low speed flow regions is formulated from a family of preconditioners popularized by Turkel [12]-[18]. This family of preconditioners include the Choi-Merkle preconditioner [20], with the particular case considered in [1]. The scheme is sometimes termed as the Roe-Turkel scheme $[2,8]$. The diagonalization of the rescaled matrix-valued dissipation must be achieved for the computation of complex flows, also because somehow an entropy fix may be used to prevent eigenvalues from approaching zero and to select the relevant physical solution satisfying the entropy condition across shocks.

By removing the iterative preconditioning, we loose the optimal conditioning but we save the essential feature of the accuracy in the incompressible limit. Without iterative preconditioning, we see that by forcing the proper scaling of the dissipation matrix, we cannot avoid the two-timescale issue, since the isentropic solution breaks up into a fast component that should disappear when the Mach number goes to zero, and a slow component solution of the incompressible equations [19]. The system then becomes very stiff and numerically difficult to solve with the explicit scheme and a time step defined by the stability condition for the fastest acoustic wave. For steady-flow problems, a robust implicit scheme must be developed to improve both the stability and the damping properties of the iterative procedure. However, we must stress that our final objective is not to develop an optimal approach specialized to handle almost incompressible flows, but only a more accurate compressible flow solver in low speed flow regions, without 
reformulating the boundary conditions and keeping the consistency in time for unsteady flows.

In the following, we shall first consider the iterative and non-iterative preconditioning following Turkel's analysis. This allows a smooth transition to the formulation of the rescaled Roe scheme and the corresponding stability condition. For steady-state problems, a robust implicit scheme has been developed in order to circumvent the severe stability bound occurring for low Mach number flows. The implicit scheme combines the introduction of a characteristic time-step matrix and under-relaxation. Preliminary results for low-speed and transonic flows are then presented. It is experienced that higher accuracy is achieved with the rescaled matrix dissipation not only in the incompressible limit but also at the stagnation point. It is especially shown in the transonic case that the known spurious entropy produced by the Roe scheme at the stagnation point is strongly reduced by the rescaling of the Roe matrix dissipation.

\section{Iterative and non-iterative preconditioning}

\subsection{Iterative preconditioning}

When considering the iterative preconditioning, the time derivatives are premultiplied by a matrix which slows down the speed of the acoustic waves down the fluid speed. Hence, at low Mach numbers all the waves will travel at the same speed and therefore, the equations will be again well conditioned. The preconditioned Euler equations for the iterative preconditioning are formulated as follows

$$
\boldsymbol{P}^{-1} \frac{\partial \boldsymbol{W}}{\partial t}=-\left(\frac{\partial \boldsymbol{F}}{\partial x}+\frac{\partial \boldsymbol{G}}{\partial y}\right)=-\left(\boldsymbol{A} \frac{\partial \boldsymbol{W}}{\partial x}+\boldsymbol{B} \frac{\partial \boldsymbol{W}}{\partial y}\right),
$$

where $\boldsymbol{P}$ is a preconditioning matrix. The expression of the Jacobian matrices $\boldsymbol{A}, \boldsymbol{B}$ depends on the choice of the variables $\boldsymbol{W}$. However, the preconditioning can be viewed as an artificial compressibility effect of the incompressible Euler equations and was first formulated for the Euler equations formulated for the primitive variables. The formulation of the preconditioner described below is the generalization of previous preconditioners due to Turkel [13], considering the independent variables $\boldsymbol{W}_{\mathbf{0}}=[p, u, v, S]^{T}$ where $p$ is the pressure $u, v$ are the velocity components and $S$ is the entropy. This leads to a non-conservative formulation for ease of presentation. The Euler equations are then formulated in quasi-linear form

$$
\frac{\partial \boldsymbol{W}_{0}}{\partial t}=-\left[\boldsymbol{A}_{\mathbf{0}} \frac{\partial \boldsymbol{W}_{\mathbf{0}}}{\partial x}+\boldsymbol{B}_{0} \frac{\partial \boldsymbol{W}_{0}}{\partial y}\right],
$$


with the corresponding Jacobian matrices in the $(x, y)$ directions

$$
\boldsymbol{A}_{\mathbf{0}}=\left(\begin{array}{cccc}
u & \rho a^{2} & 0 & 0 \\
\frac{1}{\rho} & u & 0 & 0 \\
0 & 0 & u & 0 \\
0 & 0 & 0 & u
\end{array}\right) \quad, \quad \boldsymbol{B}_{0}=\left(\begin{array}{cccc}
v & 0 & \rho a^{2} & 0 \\
0 & v & 0 & 0 \\
\frac{1}{\rho} & 0 & v & 0 \\
0 & 0 & 0 & v
\end{array}\right) .
$$

The family of preconditioners we shall consider contain a free parameter $\beta$ as formulated by Choi-Merkle and is defined as

$$
\boldsymbol{P}_{0}{ }^{-1} \frac{\partial W_{0}}{\partial t}=-\left[\boldsymbol{A}_{0} \frac{\partial \boldsymbol{W}_{0}}{\partial x}+\boldsymbol{B}_{0} \frac{\partial W_{0}}{\partial y}\right]
$$

with

$$
\boldsymbol{P}_{0}^{-1}=\left(\begin{array}{cccc}
\frac{a^{2}}{\beta^{2}} & 0 & 0 & \delta \\
\frac{\alpha u}{\rho \beta^{2}} & 1 & 0 & 0 \\
\frac{\alpha v}{\rho \beta^{2}} & 0 & 1 & 0 \\
0 & 0 & 0 & 1
\end{array}\right), \quad \boldsymbol{P}_{0}=\left(\begin{array}{cccc}
\frac{\beta^{2}}{a^{2}} & 0 & 0 & -\frac{\beta^{2}}{a^{2}} \delta \\
-\frac{\alpha u}{\rho a^{2}} & 1 & 0 & \frac{\alpha u}{\rho a^{2}} \delta \\
-\frac{\alpha v}{\rho a^{2}} & 0 & 1 & \frac{\alpha v}{\rho a^{2}} \delta \\
0 & 0 & 0 & 1
\end{array}\right) .
$$

For $\delta=0$ we get the family of preconditioners including the Weiss-Smith formulation as suggested by Turkel [14], while for $\delta=1$ and $\alpha=0$ we recover the preconditioner introduced by Choi and Merkle [20]. Setting $\alpha=0$ corresponds to the original artificial compressibility method formulated for the primitive variables while $\alpha=-1$ corresponds to the artificial compressibility method formulated for the conservatives variables [12]. In all the following the free parameter is formulated as a square of the local Mach number with

$$
\frac{\beta^{2}}{a^{2}}=\min \left(\max \left(k_{1} M^{2}, \epsilon^{2}\right), 1\right)
$$

where usually $\epsilon^{2} \simeq k_{2} M_{\infty}^{2}$. Computationally, $k_{2}$ is found to depend on the number of grid nodes near the stagnation points. For viscous flows, $\mathrm{k} 2$ also depend on $\alpha$ and may depend on the local cell Reynolds number [20]. Note that in the next sections, the preconditioning parameter homogeneous to a speed $|\beta|>0$ is introduced, with the following definition

$$
|\beta|=a \sqrt{\min \left(\max \left(k_{1} M^{2}, \epsilon^{2}\right), 1\right)} .
$$


To transition smoothly to transonic flow, we demand that $\frac{\beta^{2}}{a^{2}} \rightarrow 1$ with $\alpha=0$ as $M \rightarrow 1$. In that case the preconditioning matrix returns the identity matrix.

We consider now the steady-state problem for the preconditioned Euler equations. We reformulate system (3) as follows

$$
\frac{\partial \boldsymbol{W}_{\mathbf{0}}}{\partial t}=-\left[\left(\boldsymbol{P}_{\mathbf{0}} \boldsymbol{A}_{\mathbf{0}}\right) \frac{\partial \boldsymbol{W}_{\mathbf{0}}}{\partial x}+\left(\boldsymbol{P}_{\mathbf{0}} \boldsymbol{B}_{\mathbf{0}}\right) \frac{\partial \boldsymbol{W}_{\mathbf{0}}}{\partial y}\right]
$$

The eigenvalues of $\boldsymbol{P}_{\mathbf{0}} \boldsymbol{A}_{\mathbf{0}}$ are independent of the parameter $\delta$, with

$$
\left\{\begin{array}{l}
\lambda_{0}=u, \\
\lambda_{ \pm}=z u \pm \sqrt{(z u)^{2}+\frac{\beta^{2}}{a^{2}}\left(a^{2}-u^{2}\right),} \quad z=0.5\left(1-\alpha+\frac{\beta^{2}}{a^{2}}\right)
\end{array}\right.
$$

In a straightforward implementation of this procedure, the preconditioning algorithm can be converted from any set of variables to the appropriate conditioning matrix for the conservation variables. Let $\boldsymbol{W}_{\mathbf{1}}$ represent some set of independent variables and $\boldsymbol{W}_{\mathbf{0}}$ the solution of the preconditioned equations (7), expressed as a function of the variables $\boldsymbol{W}_{\mathbf{1}}$ with $\boldsymbol{W}_{\mathbf{0}}\left(\boldsymbol{W}_{\mathbf{1}}\right)$. Then we also have in variables $\boldsymbol{W}_{\mathbf{1}}$

$$
\frac{\partial \boldsymbol{W}_{1}}{\partial t}=-\left[\left(\boldsymbol{P}_{1} \boldsymbol{A}_{1}\right) \frac{\partial \boldsymbol{W}_{1}}{\partial x}+\left(\boldsymbol{P}_{1} \boldsymbol{B}_{1}\right) \frac{\partial \boldsymbol{W}_{1}}{\partial y}\right] .
$$

with the preconditioner and the flux Jacobian matrices obtained by similarity transformation

$$
\boldsymbol{P}_{1}=\frac{\partial W_{1}}{\partial \boldsymbol{W}_{0}} \boldsymbol{P}_{0} \frac{\partial W_{0}}{\partial W_{1}}, \quad \boldsymbol{A}_{1}=\frac{\partial W_{1}}{\partial W_{0}} \boldsymbol{A}_{0} \frac{\partial W_{0}}{\partial W_{1}}, \quad B_{1}=\frac{\partial W_{1}}{\partial W_{0}} B_{0} \frac{\partial W_{0}}{\partial W_{1}} .
$$

If $\boldsymbol{W}_{\mathbf{1}}=\boldsymbol{W}$ represents the conservative variables, with $\boldsymbol{A}=\frac{\partial \boldsymbol{F}}{\partial \boldsymbol{W}}, \boldsymbol{B}=\frac{\partial \boldsymbol{G}}{\partial \boldsymbol{W}}$, we recover the preconditioned Euler equations for the conservative variables

$$
\frac{\partial \boldsymbol{W}}{\partial t}=-\boldsymbol{P}\left(\frac{\partial \boldsymbol{F}}{\partial x}+\frac{\partial \boldsymbol{G}}{\partial y}\right)
$$

Confronting expressions (7) and (9) for the matrix coefficients, the preconditioned Jacobian matrices $\left(\boldsymbol{P}_{\mathbf{0}} \boldsymbol{A}_{\mathbf{0}}\right),\left(\boldsymbol{P}_{\mathbf{0}} \boldsymbol{B}_{\mathbf{0}}\right)$ and $\left(\boldsymbol{P}_{\mathbf{1}} \boldsymbol{A}_{\mathbf{1}}\right),\left(\boldsymbol{P}_{\mathbf{1}} \boldsymbol{B}_{\mathbf{1}}\right)$ are similar by any change of independent variables and therefore have the same eigenvalues. The same transformations apply to the rescaled Roe scheme derived in the next sections.

\subsection{Extension to time-dependent flows}

Turkel has proposed a straightforward extension of low speed preconditioning for time dependent problems, based on the dual-time stepping method [16]. The unsteady equations are then replaced by

$$
\frac{\partial \boldsymbol{W}}{\partial \tau}+\left[\frac{\partial \boldsymbol{W}}{\partial t}+\frac{\partial \boldsymbol{F}}{\partial x}+\frac{\partial \boldsymbol{G}}{\partial y}\right]=\mathbf{0}
$$


where $\tau$ is the dual time. The time derivative is discretized by some first or second-order differencing formula. A typical implicit difference scheme for the preconditioned system in pseudotime with generic artificial dissipation terms can be expressed with the following conservative formulation

$$
\Delta \boldsymbol{W}=-\Delta t^{*} \boldsymbol{P}\left[\frac{1}{\Delta t}\left(c_{0} \boldsymbol{W}^{n}+\sum_{k \in\{-1,1\}} c_{k} \boldsymbol{W}^{n+k}\right)+\left(\frac{\partial \boldsymbol{F}}{\partial x}+\frac{\partial \boldsymbol{G}}{\partial y}\right)-\frac{\partial}{\partial x}\left(\boldsymbol{P}^{-1}|\boldsymbol{P} \boldsymbol{A}| \frac{\partial \boldsymbol{W}}{\partial x}\right)-\frac{\partial}{\partial y}\left(\boldsymbol{P}^{-1}|\boldsymbol{P} \boldsymbol{B}| \frac{\partial \boldsymbol{W}}{\partial y}\right)\right],
$$

where $\Delta t^{*}$ is a pseudo time-step. The numerical treatment for the artificial dissipation is then unchanged by the introduction of the backward differencing formula considered for the approximation of the time derivative.

\subsection{Non-iterative preconditioning}

A second approach to handle both steady and time-dependent flows is described in [1]. A time-consistent method is considered with the preconditioning used as a way of controlling the effects of the artificial dissipation in case of low speed flow. The proposed method is formulated for the conservative variables by removing the pre-multiplication of the residual with the preconditioning matrix

$$
\Delta \boldsymbol{W}=-\Delta t\left[\left(\frac{\partial \boldsymbol{F}}{\partial x}+\frac{\partial \boldsymbol{G}}{\partial y}\right)-\frac{\partial}{\partial x}\left(\boldsymbol{P}^{-1}|\boldsymbol{P} \boldsymbol{A}| \frac{\partial \boldsymbol{W}}{\partial x}\right)-\frac{\partial}{\partial y}\left(\boldsymbol{P}^{-1}|\boldsymbol{P} \boldsymbol{B}| \frac{\partial \boldsymbol{W}}{\partial y}\right)\right] .
$$

We can see that system (14) is a time-consistent discretization of the non-preconditioned Euler equations based on a backward Euler approximation of the time derivative. In the framework of a steady state problem, the time step $\Delta t$ is replaced by a local time step. This non-iterative preconditioning algorithm is interpreted by Turkel as a preconditioning of the stabilization term only. Following the same idea, we may also consider the following dual time-stepping scheme for unsteady problems

$$
\Delta \boldsymbol{W}=-\Delta t^{*}\left[\frac{1}{\Delta t}\left(c_{0} \boldsymbol{W}^{n}+\sum_{k \in\{-1,1\}} c_{k} \boldsymbol{W}^{n+k}\right)+\left(\frac{\partial \boldsymbol{F}}{\partial x}+\frac{\partial \boldsymbol{G}}{\partial y}\right)-\frac{\partial}{\partial x}\left(\boldsymbol{P}^{-1}|\boldsymbol{P} \boldsymbol{A}| \frac{\partial \boldsymbol{W}}{\partial x}\right)-\frac{\partial}{\partial y}\left(\boldsymbol{P}^{-1}|\boldsymbol{P} \boldsymbol{B}| \frac{\partial \boldsymbol{W}}{\partial y}\right)\right] .
$$

We clearly see that both schemes can be interpreted as a modification of the matrix-valued dissipation scheme. Thus, we will prefer to consider the formulations (14) or (15) as a rescaling of the dissipation for low-speed flows, since the two approaches are quite different. The noniterative preconditioning achieves by construction the proper scaling of the matrix dissipation in the incompressible limit, but doesn't improve the condition number of the iterative procedure and the stability bound is drastically modified in the low-speed flow regions. Additionally formulations (14) or (15) alleviate its implementation in a CFD code since once removed the iterative 
preconditioning, there is no need to reformulate the boundary conditions using the characteristic theory or the Riemann invariants. As a final comment, for the steady-state problem or the time-dependent problem, the time or pseudo-time derivative vanishes and since the preconditioning matrix is not singular, both iterative and non-iterative preconditioning methods fall into the same steady-state or unsteady equations. At the asymptotic convergence of the iterative process it is worth noticing that in both cases the governing matrix dissipation is given by coefficients $\left(\boldsymbol{P}^{-1}|\boldsymbol{P A}|, \boldsymbol{P}^{-1}|\boldsymbol{P} \boldsymbol{B}|\right)$ in the $(x, y)$ space directions.

However, for the linear problem, the stability of the iterative preconditioning is characterized by $|\boldsymbol{P A}|$ and $|\boldsymbol{P} \boldsymbol{B}|$, while removing the pre-multiplication of the residual with $\boldsymbol{P}$, the stability must be reconsidered from matrices $\boldsymbol{P}^{-1}|\boldsymbol{P A}|$ and $\boldsymbol{P}^{-1}|\boldsymbol{P} \boldsymbol{B}|$. It becomes then essential to reformulate the Von Neumann condition by deriving algebraically the corresponding eigenspaces and eigenvalues. This can be achieved step by step as indicated in the two next sections, starting from the stream-aligned formulation of the Euler equations for the symmetrizing entropic variables.

\section{Formulation of the rescaled Roe scheme}

\subsection{One-dimensional framework}

\subsubsection{Iterative preconditioning}

In order to investigate the limit to incompressible equations as the Mach number goes to zero, we follow Turkel [17] and explicitly describe the scheme for the one-dimensional equations, including a second-order artificial viscosity term. For the equations termed in entropic variables, we shall consider first a streamwise two-dimensional coordinate system with the $x$-axis aligned to the flow. Then setting $\frac{\partial v}{\partial y}=0, \frac{\partial p}{\partial y}=0$ in the basic system (1) and taking as independent variables for the pressure $d \Phi=\frac{d p}{\rho a}$ and $d S=\frac{d p-a^{2} d \rho}{\rho a}$ proportional to the entropy for the symmetrizing 
variables, system (1) can be recast as follows

$$
\left\{\begin{array}{l}
\frac{\partial \Phi}{\partial t}+u \frac{\partial \Phi}{\partial x}+a \frac{\partial u}{\partial x}=0 \\
\frac{\partial u}{\partial t}+a \frac{\partial \Phi}{\partial x}+u \frac{\partial u}{\partial x}=0 \\
\frac{\partial v}{\partial t}+u \frac{\partial v}{\partial x}=0 \\
\frac{\partial S}{\partial t}+u \frac{\partial S}{\partial x}=0
\end{array}\right.
$$

The equations for $v$ and $S$ decouple and so we shall consider the two acoustic equations. Let introduce some artificial dissipation terms with constant coefficients. We then get the following scheme

$$
\left\{\begin{array}{l}
\frac{\partial \Phi}{\partial t}+u \frac{\partial \Phi}{\partial x}+a \frac{\partial u}{\partial x}=Q_{1,1} \frac{\partial^{2} \Phi}{\partial x^{2}}+Q_{1,2} \frac{\partial^{2} u}{\partial x^{2}} \\
\frac{\partial u}{\partial t}+a \frac{\partial \Phi}{\partial x}+u \frac{\partial u}{\partial x}=Q_{2,1} \frac{\partial^{2} \Phi^{2}}{\partial x^{2}}+Q_{2,2} \frac{\partial^{2} u}{\partial x^{2}}
\end{array}\right.
$$

Let $\boldsymbol{d} \tilde{\boldsymbol{W}}_{\mathbf{0}}=[d \Phi, d u]^{T}$. In matrix form, the previous system reads

$$
\frac{\partial \tilde{\boldsymbol{W}}_{\mathbf{0}}}{\partial t}+\tilde{\boldsymbol{A}}_{\mathbf{0}} \frac{\partial \tilde{\boldsymbol{W}}_{\mathbf{0}}}{\partial x}=\boldsymbol{Q} \frac{\partial^{2} \tilde{\boldsymbol{W}}_{\mathbf{0}}}{\partial x^{2}}
$$

with the symmetric Jacobian matrix

$$
\tilde{A}_{\mathbf{0}}=\left(\begin{array}{cc}
u & a \\
& \\
a & u
\end{array}\right) .
$$

The matrix $\tilde{A}_{0}$ has the two eigenvalues $\lambda_{ \pm}=u \pm a$ corresponding to the acoustic waves. The limit to incompressible equations for the scheme (17) has been investigated in [17, 18]. From now on, all quantities $\rho, u, v, p, S$ are nondimensionalized by some reference quantities $\rho_{r}, u_{r}, p_{r}=$ $\rho_{r} u_{r}^{2}, S_{r}=r_{r}$, where $r_{r}$ is the reference perfect gas constant. Let introduce $\epsilon=\frac{u_{r}}{a_{r}}=M$. In the incompressible limit, the fluid is assumed isentropic. Then

$$
p=\frac{\rho^{\gamma}}{\gamma \epsilon^{2}} \simeq O\left(\frac{1}{M^{2}}\right), \quad a=\frac{\rho^{\frac{\gamma-1}{2}}}{\epsilon} \simeq O\left(\frac{1}{M}\right) \quad \text { as } \quad M \rightarrow 0 .
$$


Within a formal asymptotic analysis, the mesh size being kept constant, it was found that in the incompressible limit, the proper scaling of the matrix-valued dissipation requires

$$
Q_{1,1}=O\left(\frac{1}{M^{2}}\right), \quad Q_{1,2}=O\left(\frac{1}{M}\right), \quad Q_{2,1}=O\left(\frac{1}{M}\right), \quad Q_{2,2}=O(1) .
$$

For the system (17), we assume that the artificial dissipation is some function of the matrix $\left|\tilde{A}_{0}\right|$. In the case of subsonic flow $|u| \leq a$, we have the following diagonalization

$$
\left|\tilde{A}_{\mathbf{0}}\right|=\left(\begin{array}{cc}
a & a \\
a & -a
\end{array}\right)\left(\begin{array}{cc}
a+u & 0 \\
0 & a-u
\end{array}\right)\left(\begin{array}{cc}
\frac{1}{2 a} & \frac{1}{2 a} \\
\frac{1}{2 a} & -\frac{1}{2 a}
\end{array}\right)=\left(\begin{array}{cc}
a & u \\
& \\
u & a
\end{array}\right) .
$$

Thus, as $M \rightarrow 0$ :

$$
\left|\tilde{A}_{0}\right| \simeq\left(\begin{array}{cc}
O\left(\frac{1}{M}\right) & O(1) \\
O(1) & O\left(\frac{1}{M}\right)
\end{array}\right) .
$$

This implies that the momentum equation has too much artificial viscosity, while the the divergence equation has too little viscosity which will cause oscillations. For the system in variables $d \tilde{W}_{0}$, the preconditioner becomes

$$
\tilde{\boldsymbol{P}}_{\mathbf{0}}^{-1}=\left(\begin{array}{cc}
\frac{a^{2}}{\beta^{2}} & 0 \\
\frac{\alpha u a}{\beta^{2}} & 1
\end{array}\right) \quad, \quad \tilde{\boldsymbol{P}}_{\mathbf{0}}=\left(\begin{array}{cc}
\frac{\beta^{2}}{a^{2}} & 0 \\
-\frac{\alpha u}{a} & 1
\end{array}\right) .
$$

The preconditioned Jacobian matrix

$$
\tilde{\boldsymbol{P}}_{\mathbf{0}} \tilde{\boldsymbol{A}}_{\mathbf{0}}=\left(\begin{array}{cc}
\frac{\beta^{2}}{a^{2}} u & \frac{\beta^{2}}{a} \\
a\left(1-\alpha \frac{u^{2}}{a^{2}}\right) & (1-\alpha) u
\end{array}\right)
$$

has eigenvalues $\lambda_{ \pm}$given by (8). We have the following diagonalization

$$
\left|\tilde{\boldsymbol{P}}_{\mathbf{0}} \tilde{A}_{\mathbf{0}}\right|=\tilde{\boldsymbol{R}}|\boldsymbol{\Lambda}| \tilde{\boldsymbol{R}}^{-1},
$$


where $|\boldsymbol{\Lambda}|=\operatorname{diag}\left(\left|\lambda_{+}\right|, \mid \lambda_{1}\right), \boldsymbol{R}$ and $\tilde{\boldsymbol{R}}^{-1}$ are respectively the matrices of the right and left eigenvectors

$$
\tilde{\boldsymbol{R}}=\left(\begin{array}{cc}
\frac{\beta^{2}}{a} & \frac{\beta^{2}}{a} \\
\lambda_{+}-\frac{\beta^{2}}{a^{2}} u & \lambda_{-}-\frac{\beta^{2}}{a^{2}} u
\end{array}\right), \quad \tilde{\boldsymbol{R}}^{-1}=\frac{1}{\left(\lambda_{+}-\lambda_{-}\right)}\left(\begin{array}{cc}
\frac{a}{\beta^{2}}\left[\lambda_{+}-(1-\alpha) u\right] & 1 \\
-\frac{a}{\beta^{2}}\left[\lambda_{-}-(1-\alpha) u\right] & -1
\end{array}\right) .
$$

\subsubsection{Rescaled Roe scheme}

By removing the iterative preconditioning, we introduce the rescaled Roe scheme

$$
\frac{\partial \tilde{\boldsymbol{W}}_{\mathbf{0}}}{\partial t}+\tilde{\boldsymbol{A}}_{\mathbf{0}} \frac{\partial \tilde{\boldsymbol{W}}_{\mathbf{0}}}{\partial x}=\tilde{\boldsymbol{Q}}_{\mathbf{0}} \frac{\partial^{2} \tilde{\boldsymbol{W}}_{\mathbf{0}}}{\partial x^{2}},
$$

with the matrix-valued dissipation

$$
\tilde{\boldsymbol{Q}}_{\mathbf{0}}=\tilde{\boldsymbol{P}}_{\mathbf{0}}^{-1}\left|\tilde{\boldsymbol{P}}_{\mathbf{0}} \tilde{\boldsymbol{A}}_{\mathbf{0}}\right|=\tilde{\boldsymbol{P}}_{\mathbf{0}}^{-1} \tilde{\boldsymbol{R}}|\boldsymbol{\Lambda}| \tilde{\boldsymbol{R}}^{-1}
$$

In the following, we will assume the flow subsonic. Let $\chi=(z u)^{2}+\frac{\beta^{2}}{a^{2}}\left(a^{2}-u^{2}\right)$. We always have $x>0$ and

$$
\chi \geq(z u)^{2} \Rightarrow(z u-\sqrt{\chi})(z u+\sqrt{\chi}) \leq 0 .
$$

Hence, eigenvalues $\lambda_{+}$and $\lambda_{-}$have opposite signs for a subsonic flow with

$$
\begin{aligned}
& \text { if } u \leq 0 \rightarrow \lambda_{-} \leq 0 \Rightarrow \lambda_{+} \geq 0, \\
& \text { if } u \geq 0 \rightarrow \lambda_{+} \geq 0 \Rightarrow \lambda_{-} \leq 0 .
\end{aligned}
$$

Thus $\left|\lambda_{+}\right|=\lambda_{+}$and $\left|\lambda_{-}\right|=-\lambda_{-}$and we get an explicit expression of the dissipation matrix

$$
\tilde{\boldsymbol{P}}_{\mathbf{0}}^{-1}\left|\tilde{\boldsymbol{P}}_{\mathbf{0}} \tilde{\boldsymbol{A}}_{\mathbf{0}}\right|=\frac{1}{\sqrt{\chi}}\left(\begin{array}{cc}
{\left[z M^{2}+\left(1-M^{2}\right)\right] a^{2}} & z \text { и } а \\
{\left[z+\alpha\left(1-M^{2}\right)\right] u a} & {\left[z M^{2}+\frac{\beta^{2}}{a^{2}}\left(1-M^{2}\right)\right] a^{2}}
\end{array}\right),
$$

where $M=\frac{|u|}{a}$. Note that for the two acoustic equations

$$
\tilde{\boldsymbol{P}}_{\mathbf{0}}^{-1}\left|\tilde{\boldsymbol{P}}_{\mathbf{0}} \tilde{\boldsymbol{A}}_{\mathbf{0}}\right|=\frac{1}{\sqrt{\chi}}\left[z u \tilde{\boldsymbol{A}}_{\mathbf{0}}+\frac{\beta^{2}}{a^{2}}\left(a^{2}-u^{2}\right) \tilde{\boldsymbol{P}}_{\mathbf{0}}^{-1}\right]
$$

as a consequence of the Cayley-Hamilton theorem. Therefore the dissipation matrix can be interpreted as a weighted average between the Jacobian matrix and the preconditioner. At a sonic point the second coefficient vanishes and it can be easily verified that for both possible values 
$u= \pm a$ the rescaled dissipation matrix returns $\left|\tilde{\boldsymbol{A}}_{\mathbf{0}}\right|$ for $\alpha=0$. Let be $Q_{i j}$ the coefficients of matrix (29). In the limit to the incompressible equations, assuming $M_{\infty}<<1$ and $\beta^{2} \simeq u^{2}=O(1)$,we successively have

$$
\frac{\beta^{2}}{a^{2}} \simeq \frac{u^{2}}{a^{2}}=O\left(M^{2}\right), \quad \chi=z^{2} u^{2}+\beta^{2}\left(1-M^{2}\right) \simeq u^{2}=O(1),
$$

and

$$
\begin{array}{ll}
Q_{11}=\frac{1}{\sqrt{\chi}}\left[z M^{2}+\left(1-M^{2}\right)\right] a^{2} \simeq \frac{a^{2}}{|u|}=O\left(\frac{1}{M^{2}}\right), & Q_{12}=\frac{1}{\sqrt{\chi}} z u a \simeq a=O\left(\frac{1}{M}\right) \\
Q_{21}=\frac{1}{\sqrt{\chi}}\left[z+\alpha\left(1-M^{2}\right)\right] u a \simeq a=O\left(\frac{1}{M}\right), & Q_{22}=\frac{1}{\sqrt{\chi}}\left[z M^{2}+\frac{\beta^{2}}{a^{2}}\left(1-M^{2}\right)\right] a^{2} \simeq|u|=O(1) .
\end{array}
$$

Then, as $M \rightarrow 0$

$$
\tilde{\boldsymbol{P}}_{\mathbf{0}}^{-1}\left|\tilde{\boldsymbol{P}}_{\mathbf{0}} \tilde{\boldsymbol{A}}_{\mathbf{0}}\right| \simeq\left(\begin{array}{cc}
O\left(\frac{1}{M^{2}}\right) & O\left(\frac{1}{M}\right) \\
O\left(\frac{1}{M}\right) & O(1)
\end{array}\right),
$$

and the scheme gives the correct asymptotic order for the artificial dissipation terms. It is worth noticing that the the rescaling (30) corresponds to a net increase of numerical dissipation in the pressure equation by a factor $\frac{1}{M}$ compared to the unpreconditioned scheme (23) whereas in the equation for the stream-aligned velocity, the coefficients of the stabilization terms are consistently rescaled by the preconditioner.

We also see that matrix $\tilde{\boldsymbol{P}}_{\mathbf{0}}^{-1}\left|\tilde{\boldsymbol{P}}_{\mathbf{0}} \tilde{\boldsymbol{A}}_{\mathbf{0}}\right|$ has two real eigenvalues given by

$$
\mu_{ \pm}=\frac{a}{2 \sqrt{\tilde{\chi}}}\left[2 z+\alpha\left(1-M^{2}\right) \pm \sqrt{\Delta}\right]
$$

with

$$
\Delta=\left(1-\frac{\beta^{2}}{a^{2}}\right)^{2}\left(1-M^{2}\right)^{2}+4 z\left[z+\alpha\left(1-M^{2}\right)\right] M^{2}, \quad \tilde{\chi}=z^{2} M^{2}+\frac{\beta^{2}}{a^{2}}\left(1-M^{2}\right) .
$$

We find that both eigenvalues $\mu_{ \pm}$are positive as long as the flow is subsonic with

$$
\mu_{+} \mu_{-}=Q_{11} Q_{22}-Q_{12} Q_{21}=\operatorname{det}\left(\tilde{\boldsymbol{P}}_{\mathbf{0}}^{-1}\left|\tilde{\boldsymbol{P}}_{\mathbf{0}} \tilde{\boldsymbol{A}}_{\mathbf{0}}\right|\right)
$$

and

$$
\operatorname{det}\left(\tilde{\boldsymbol{P}}_{\mathbf{0}}{ }^{-1}\left|\tilde{\boldsymbol{P}}_{\mathbf{0}} \tilde{\boldsymbol{A}}_{\mathbf{0}}\right|\right)=\frac{\left[\left(z-\frac{\beta^{2}}{a^{2}}\right)^{2}-\alpha \frac{\beta^{2}}{a^{2}}\right] u^{2}+\beta^{2}}{\left[z^{2}-\frac{\beta^{2}}{a^{2}}\right] u^{2}+\beta^{2}}\left(a^{2}-u^{2}\right)=a^{2}-u^{2}=\operatorname{det}\left(\left|\tilde{\boldsymbol{A}}_{\mathbf{0}}\right|\right)>0 .
$$


Note that rescaled matrix dissipation is symmetric for $\alpha=0$ and therefore is positive definite with the two positive eigenvalues for in case of subsonic flow. This property is lost by any change of variables that does not preserve the symmetry of $\tilde{\boldsymbol{P}}_{\mathbf{0}}{ }^{-1}\left|\tilde{\boldsymbol{P}}_{\mathbf{0}} \tilde{\boldsymbol{A}}_{\mathbf{0}}\right|$. In the incompressible limit, the eigenvalues behave as

$$
\mu_{ \pm} \simeq \frac{a^{2}}{2|\beta|}\left[1+\frac{\beta^{2}}{a^{2}} \pm\left|1-\frac{\beta^{2}}{a^{2}}\right|\right]
$$

Since $\frac{\beta^{2}}{a^{2}} \leq 1$

$$
\mu_{+} \simeq \frac{a^{2}}{|\beta|}=O\left(\frac{1}{M^{2}}\right), \quad \mu_{-} \simeq|\beta|=O(1)
$$

Thus, in the asymptotic limit acoustics waves associated to $\mu_{+}$will travel at infinite speed while acoustic waves associated to $\mu_{-}$are slowed down to the flow velocity. This is a general behaviour of the eigenvalues $\mu_{ \pm}$in the low speed limit, because (30) holds necessarily with the coefficients $Q_{i j}$ for a proper scaling of the artificial dissipation, whatever the definition of the preconditioner $\tilde{\boldsymbol{P}}_{\mathbf{0}}$. This raises the well known singularity of the two time-scale problem. The optimal conditioning of the iterative preconditioning is lost and is even worse that the original system. The behaviour of eigenvalues $\mu_{ \pm}$in the low Mach number limit will become critical for the stability of the rescaled Roe scheme and a CFL condition must be formulated accordingly. For the other extreme case, at a sonic line, for $\alpha=0$, we see that we recover the values of the unpreconditioned system

$$
\mu_{+}=2 a, \quad \mu_{-}=0
$$

When the preconditioning is not activated, setting $\alpha=0$ and $\frac{\beta^{2}}{a^{2}}=1$ in expressions (31) gives $\mu_{ \pm}=a(1 \pm M)=a \pm|u|$, the eigenvalues of $\left|\tilde{\boldsymbol{A}}_{\mathbf{0}}\right|$ recast for the velocity module.

We can also easily derive the following diagonalization of the rescaled matrix dissipation

$$
\tilde{\boldsymbol{P}}_{\mathbf{0}}{ }^{-1}\left|\tilde{\boldsymbol{P}}_{\mathbf{0}} \tilde{A}_{\mathbf{0}}\right|=\tilde{\boldsymbol{R}}_{\mathbf{0}} \tilde{\Lambda} \tilde{\boldsymbol{R}}_{\mathbf{0}}{ }^{-1}
$$

where $\tilde{\boldsymbol{\Lambda}}=\operatorname{diag}\left(\mu_{+}, \mu_{-}\right), \tilde{\boldsymbol{R}}_{\mathbf{0}}$ is the matrix of the right eigenvectors for the acoustic eigenvalues $\mu_{ \pm}$and $\tilde{\boldsymbol{R}}_{\mathbf{0}}{ }^{-1}$ is obtained by direct inversion of $\tilde{\boldsymbol{R}}_{\mathbf{0}}$

$$
\tilde{\boldsymbol{R}}_{\mathbf{0}}=\left(\begin{array}{cc}
{\left[\mu_{+}-Q_{22}\right]} & Q_{12} \\
Q_{21} & {\left[\mu_{-}-Q_{11}\right]}
\end{array}\right), \quad \tilde{\boldsymbol{R}}_{\mathbf{0}}^{-1}=\frac{1}{\operatorname{det}\left(\tilde{\boldsymbol{R}}_{\mathbf{0}}\right)}\left(\begin{array}{cc}
{\left[\mu_{-}-Q_{11}\right]} & -Q_{12} \\
-Q_{21} & {\left[\mu_{+}-Q_{22}\right]}
\end{array}\right),
$$


with the determinant of matrix $\tilde{\boldsymbol{R}}_{\mathbf{0}}$ given by

$$
\begin{aligned}
\operatorname{det}\left(\tilde{\boldsymbol{R}}_{\mathbf{0}}\right) & =\left[\mu_{-}-Q_{11}\right]\left[\mu_{+}-Q_{22}\right]-Q_{12} Q_{21}=\mu_{+}\left[\mu_{-}-Q_{11}\right]+\mu_{-}\left[\mu_{+}-Q_{22}\right] \\
& =\left[\mu_{+}-Q_{22}\right]\left[\mu_{-}-\mu_{+}\right]=\left[Q_{11}-\mu_{-}\right]\left[\mu_{-}-\mu_{+}\right] .
\end{aligned}
$$

At a stagnation point, $Q_{12}=0, Q_{21}=0$, and

$$
\mu_{+}=Q_{11}=\frac{a^{2}}{|\beta|}, \quad \mu_{-}=Q_{22}=|\beta|, \quad \mu_{+}-\mu_{-}=|\beta|\left(\frac{a^{2}}{\beta^{2}}-1\right) .
$$

Thus with $\mu_{+}-\mu_{-} \neq 0$, we always have $\mu_{-}-Q_{11} \neq 0, \mu_{+}-Q_{22} \neq 0$, and the decomposition (32) can never become singular in the range $|u|<a$. Additionally, with $\alpha=0$, since by construction $\mu_{+}+\mu_{-}=Q_{11}+Q_{22}$, we have

$$
\left(\mu_{+}-Q_{22}\right) Q_{12}+\left(\mu_{-}-Q_{11}\right) Q_{21}=0 \quad \text { with } \quad Q_{12}=Q_{21},
$$

and we can see that the right and left eigenvectors are orthogonal. In this special case

$$
\operatorname{det}\left(\tilde{\boldsymbol{R}}_{\mathbf{0}}\right)=-\left[\left(\mu_{+}-Q_{22}\right)^{2}+Q_{12}^{2}\right] .
$$

Then defining

$$
\tilde{\boldsymbol{M}}_{\mathbf{0}}=\frac{1}{\sqrt{\left(\mu_{+}-Q_{22}\right)^{2}+Q_{12}^{2}}} \tilde{\boldsymbol{R}}_{\mathbf{0}}
$$

the symmetric matrix $\tilde{\boldsymbol{P}}_{\mathbf{0}}^{-1}\left|\tilde{\boldsymbol{P}}_{\mathbf{0}} \tilde{A}_{\mathbf{0}}\right|$ is diagonalized by the following unitary congruence

$$
\tilde{\boldsymbol{P}}_{0}^{-1}\left|\tilde{\boldsymbol{P}}_{0} \tilde{A}_{0}\right|=\tilde{M}_{0} \tilde{\Lambda} \tilde{M}_{0}^{T}
$$

\subsection{Multi-dimensional extension}

\subsubsection{Iterative preconditioning}

We derive the multidimensional extension of the previous analysis, assuming the flow subsonic. Let be $\boldsymbol{d} \tilde{\boldsymbol{W}}_{\mathbf{0}}=[d \Phi, d u, d v, d S]^{T}$ the set of entropy variables for the two-dimensional Euler equations. In matrix form, the two-dimensional scheme reads

$$
\frac{\partial \tilde{\boldsymbol{W}}_{\mathbf{0}}}{\partial t}+\tilde{\boldsymbol{A}}_{\mathbf{0}} \frac{\partial \tilde{\boldsymbol{W}}_{\mathbf{0}}}{\partial x}+\tilde{\boldsymbol{B}}_{0} \frac{\partial \tilde{\boldsymbol{W}}_{\mathbf{0}}}{\partial y}=\boldsymbol{Q}_{x} \frac{\partial^{2} \tilde{\boldsymbol{W}}_{\mathbf{0}}}{\partial x^{2}}+\boldsymbol{Q}_{y} \frac{\partial^{2} \tilde{\boldsymbol{W}}_{\mathbf{0}}}{\partial y^{2}},
$$


with the expressions of the Jacobian matrix in the $(x, y)$ directions

$$
\tilde{\boldsymbol{A}}_{\mathbf{0}}=\left(\begin{array}{cccc}
u & a & 0 & 0 \\
a & u & 0 & 0 \\
0 & 0 & u & 0 \\
0 & 0 & 0 & u
\end{array}\right), \quad \tilde{\boldsymbol{B}}_{\mathbf{0}}=\left(\begin{array}{cccc}
v & 0 & a & 0 \\
0 & v & 0 & 0 \\
a & 0 & v & 0 \\
0 & 0 & 0 & v
\end{array}\right),
$$

and $\boldsymbol{Q}_{x}$ and $\boldsymbol{Q}_{y}$ are some matrix-valued artificial dissipation coefficients in the $(x, y)$ directions. For a general direction $\boldsymbol{n}=\left[n_{x}, n_{y}\right]^{T}$, we will consider a matrix-valued dissipation in the general form $\boldsymbol{Q}(\boldsymbol{n})=n_{x} \boldsymbol{Q}_{\boldsymbol{x}}+n_{y} \boldsymbol{Q}_{\boldsymbol{y}}$. As the Mach number goes to zero, a proper scaling of system (37) is achieved when the coefficients of the matrix-valued dissipation satisfy [17, 18]

$$
\boldsymbol{Q}(\boldsymbol{n}) \simeq\left(\begin{array}{cccc}
O\left(\frac{1}{M^{2}}\right) & O\left(\frac{1}{M}\right) & O\left(\frac{1}{M}\right) & 0 \\
O\left(\frac{1}{M}\right) & O(1) & O(1) & 0 \\
O\left(\frac{1}{M}\right) & O(1) & O(1) & 0 \\
0 & 0 & 0 & O(1)
\end{array}\right) \quad \text { as } \quad M \longrightarrow 0 .
$$

A standard dissipation for the scheme (37) will be some function of the Jacobian matrix $\tilde{A}_{\mathbf{0}}(\boldsymbol{n})=$ $n_{x} \tilde{\boldsymbol{A}}_{\mathbf{0}}+n_{y} \tilde{\boldsymbol{B}}_{\mathbf{0}}$. Note that we can always formulate $\tilde{\boldsymbol{A}}_{\mathbf{0}}(\boldsymbol{n})$ for any unitary vector $\boldsymbol{n}_{\mathbf{1}}=\frac{\boldsymbol{n}}{|\boldsymbol{n}|}$ since $\tilde{A}_{0}(n)=\tilde{A}_{0}\left(n_{1}\right)|n|$ independently of the choice of variables. This is obviously also the case for the dissipation matrices $\left|\tilde{\boldsymbol{A}}_{\mathbf{0}}(\boldsymbol{n})\right|$ and $\tilde{\boldsymbol{P}}_{\mathbf{0}}{ }^{-1}\left|\tilde{\boldsymbol{P}}_{\mathbf{0}} \tilde{\boldsymbol{A}}_{\mathbf{0}}(\boldsymbol{n})\right|$ that we will consider in the following. In the case of the Roe scheme, the matrix-valued dissipation is characterized by $\left|\tilde{A}_{\mathbf{0}}(\boldsymbol{n})\right|$. The matrix $\tilde{A}_{\mathbf{0}}(\boldsymbol{n})$ has eigenvalues $\lambda_{ \pm}=q \pm a|\boldsymbol{n}|$ and double $\lambda_{0}=q$, with $q=u n_{x}+v n_{y}$. Assuming subsonic flow, we have $|q| \leq a|\boldsymbol{n}|$ and $\left|\lambda_{+}\right|=a|\boldsymbol{n}|+q,\left|\lambda_{-}\right|=a|\boldsymbol{n}|-q$. Hence

$$
|\Lambda|=\operatorname{diag}(a|\boldsymbol{n}|+q, a|\boldsymbol{n}|-q,|q|,|q|)
$$


Thus, we get the explicit expression

$$
\left|\tilde{\boldsymbol{A}}_{\mathbf{0}}(\boldsymbol{n})\right|=\left(\begin{array}{cccc}
a|\boldsymbol{n}| & \frac{n_{x}}{|\boldsymbol{n}|} q & \frac{n_{y}}{|\boldsymbol{n}|} q & 0 \\
\frac{n_{x}}{|\boldsymbol{n}|} q & \frac{a}{|\boldsymbol{n}|}\left(n_{x}^{2}+n_{y}^{2} M_{n}\right) & \frac{n_{x} n_{y}}{|\boldsymbol{n}|} a\left(1-M_{n}\right) & 0 \\
\frac{n_{y}}{|\boldsymbol{n}|} q & \frac{n_{x} n_{y}}{|\boldsymbol{n}|} a\left(1-M_{n}\right) & \frac{a}{|\boldsymbol{n}|}\left(n_{y}^{2}+n_{x}^{2} M_{n}\right) & 0 \\
0 & 0 & 0 & |q|
\end{array}\right),
$$

where $M_{n}=\frac{|q|}{a|\boldsymbol{n}|}$ is the directional Mach number. Let $M=\frac{\sqrt{u^{2}+v^{2}}}{a}$ be the local Mach number. It is actually important in the following to differentiate the local Mach number defining the preconditioning parameter $\frac{\beta^{2}}{a^{2}}$ and the directional (normal) Mach number arising in the formulation of the Jacobian matrices. We have $M_{n} \leq M$. Then as $M \longrightarrow 0, M_{n} \longrightarrow 0$ and

$$
\left|\tilde{A}_{\mathbf{0}}(\boldsymbol{n})\right| \simeq\left(\begin{array}{cccc}
O\left(\frac{1}{M}\right) & O(1) & O(1) & 0 \\
O(1) & O\left(\frac{1}{M}\right) & O\left(\frac{1}{M}\right) & 0 \\
O(1) & O\left(\frac{1}{M}\right) & O\left(\frac{1}{M}\right) & 0 \\
0 & 0 & 0 & O(1)
\end{array}\right) \quad \text { as } M \longrightarrow 0 .
$$

This is to be compared to result (38). As for the one-dimensional case, we can see that without preconditioning in the incompressible limit, the pressure equation has to little numerical viscosity while the momentum equation has too much numerical viscosity. We can see that the eigenvalues of the preconditioned matrix $\tilde{\boldsymbol{P}}_{\mathbf{0}} \tilde{\boldsymbol{A}}_{\mathbf{0}}$ are independent of the parameter $\delta$ in the preconditioner. This is also true for the rescaled matrix dissipation $\tilde{\boldsymbol{P}}_{\mathbf{0}}{ }^{-1}\left|\tilde{\boldsymbol{P}}_{\mathbf{0}} \tilde{\boldsymbol{A}}_{\mathbf{0}}\right|$. Thus, in the following, we will set $\delta=0$ in the family of preconditioners, since we are basically interested in finding out a stability criteria for the rescaled Roe scheme.

For the set of variables $d \tilde{W}_{\mathbf{0}}$, the preconditioning matrix in the two-dimensional case is then 
given by

$$
\tilde{\boldsymbol{P}}_{\mathbf{0}}^{-1}=\left(\begin{array}{cccc}
\frac{a^{2}}{\beta^{2}} & 0 & 0 & 0 \\
\frac{\alpha u a}{\beta^{2}} & 1 & 0 & 0 \\
\frac{\alpha v a}{\beta^{2}} & 0 & 1 & 0 \\
0 & 0 & 0 & 1
\end{array}\right), \quad \tilde{\boldsymbol{P}}_{\mathbf{0}}=\left(\begin{array}{cccc}
\frac{\beta^{2}}{a^{2}} & 0 & 0 & 0 \\
-\frac{\alpha u}{a} & 1 & 0 & 0 \\
-\frac{\alpha v}{a} & 0 & 1 & 0 \\
0 & 0 & 0 & 1
\end{array}\right)
$$

In direction $\boldsymbol{n}$, the Jacobian matrix of the preconditioned system reads

$$
\tilde{\boldsymbol{P}}_{\mathbf{0}} \tilde{\boldsymbol{A}}_{\mathbf{0}}(\boldsymbol{n})=\left(\begin{array}{cccc}
\frac{\beta^{2}}{a^{2}} q & \frac{\beta^{2}}{a} n_{x} & \frac{\beta^{2}}{a} n_{y} & 0 \\
a\left(n_{x}-\frac{\alpha u q}{a^{2}}\right) & q-\alpha u n_{x} & -\alpha u n_{y} & 0 \\
a\left(n_{y}-\frac{\alpha v q}{a^{2}}\right) & -\alpha v n_{x} & q-\alpha v n_{y} & 0 \\
0 & 0 & 0 & q
\end{array}\right)
$$

We have the following diagonalization [15]

$$
\left|\tilde{\boldsymbol{P}}_{\mathbf{0}} \tilde{A}_{\mathbf{0}}(\boldsymbol{n})\right|=\tilde{\boldsymbol{R}}|\Lambda| \tilde{\boldsymbol{R}}^{-1},
$$

with eigenvalues

$$
\left\{\begin{array}{l}
\lambda_{0}=q, \\
\lambda_{ \pm}=z q \pm \sqrt{(z q)^{2}+\frac{\beta^{2}}{a^{2}}\left(a^{2}|\boldsymbol{n}|^{2}-q^{2}\right)} .
\end{array}\right.
$$


The matrix of the right eigenvectors given by

$$
\tilde{\boldsymbol{R}}=\left(\begin{array}{cccc}
\frac{\beta^{2}}{a} & \frac{\beta^{2}}{a} & 0 & 0 \\
\frac{\beta^{2} n_{x}-\alpha u \lambda_{+}}{\lambda_{+}-q} & \frac{\beta^{2} n_{x}-\alpha u \lambda_{-}}{\lambda_{-}-q} & -n_{y} & 0 \\
\frac{\beta^{2} n_{y}-\alpha v \lambda_{+}}{\lambda_{+}-q} & \frac{\beta^{2} n_{y}-\alpha v \lambda_{-}}{\lambda_{-}-q} & n_{x} & 0 \\
0 & 0 & 0 & 1
\end{array}\right),
$$

and the matrix of the left eigenvectors can be expressed as follows

$$
\tilde{\boldsymbol{R}}^{-1}=\left(\begin{array}{cccc}
\frac{a}{\beta^{2}} \frac{\left(\lambda_{+}-(1-\alpha) q\right)}{\lambda_{+}-\lambda_{-}} & \frac{n_{x}}{\lambda_{+}-\lambda_{-}} & \frac{n_{y}}{\lambda_{+}-\lambda_{-}} & 0 \\
-\frac{a}{\beta^{2}} \frac{\left(\lambda_{-}-(1-\alpha) q\right)}{\lambda_{+}-\lambda_{-}} & -\frac{n_{x}}{\lambda_{+}-\lambda_{-}} & -\frac{n_{y}}{\lambda_{+}-\lambda_{-}} & 0 \\
\alpha a \frac{\left(u n_{y}-v n_{x}\right)}{\left(\lambda_{+}-q\right)\left(\lambda_{-}-q\right)} & \frac{\left(\beta^{2} n_{y}-\alpha v q\right)}{\left(\lambda_{+}-q\right)\left(\lambda_{-}-q\right)} & -\frac{\left(\beta^{2} n_{x}-\alpha u q\right)}{\left(\lambda_{+}-q\right)\left(\lambda_{-}-q\right)} & 0 \\
0 & 0 & 0 & 1
\end{array}\right)
$$

\subsubsection{Rescaled Roe scheme}

We now consider the non-iterative preconditioning scheme

$$
\frac{\partial \tilde{\boldsymbol{W}}_{\mathbf{0}}}{\partial t}+\tilde{\boldsymbol{A}}_{\mathbf{0}} \frac{\partial \tilde{\boldsymbol{W}}_{\mathbf{0}}}{\partial x}+\tilde{\boldsymbol{B}}_{\mathbf{0}} \frac{\partial \tilde{\boldsymbol{W}}_{\mathbf{0}}}{\partial y}=\tilde{\boldsymbol{P}}_{\mathbf{0}}^{-1}\left|\tilde{\boldsymbol{P}}_{\mathbf{0}} \tilde{\boldsymbol{A}}_{\mathbf{0}}\right| \frac{\partial^{2} \tilde{\boldsymbol{W}}_{\mathbf{0}}}{\partial x^{2}}+\tilde{\boldsymbol{P}}_{\mathbf{0}}^{-1}\left|\tilde{\boldsymbol{P}}_{\mathbf{0}} \tilde{\boldsymbol{B}}_{\mathbf{0}}\right| \frac{\partial^{2} \tilde{\boldsymbol{W}}_{\mathbf{0}}}{\partial y^{2}} .
$$

In case of subsonic flow, we know the sign of the two acoustic eigenvalues, with $\lambda_{+} \geq 0$ and $\lambda_{-} \leq 0$. Therefore

$$
|\Lambda|=\operatorname{diag}\left(\lambda_{+},-\lambda_{-},|q|,|q|\right)
$$

We proceed as for the one-dimensional case to find an explicit expression of the matrix valued dissipation

$$
\tilde{\boldsymbol{P}}_{\mathbf{0}}^{-1}\left|\tilde{\boldsymbol{P}}_{\mathbf{0}} \tilde{\boldsymbol{A}}_{\mathbf{0}}(\boldsymbol{n})\right|=\tilde{\boldsymbol{P}}_{\mathbf{0}}^{-1}|\tilde{\boldsymbol{R}}| \Lambda \mid \tilde{\boldsymbol{R}}^{-1}
$$


Let first introduce the following coefficients

$$
\begin{array}{ll}
Q_{11}=\frac{1}{\sqrt{\chi}}|\boldsymbol{n}|^{2}\left[z M_{n}^{2}+\left(1-M_{n}^{2}\right)\right] a^{2}, & Q_{12}=\frac{1}{\sqrt{\chi}} z q a, \\
Q_{21}=\frac{1}{\sqrt{\chi}}|\boldsymbol{n}|^{2}\left[z+\alpha\left(1-M_{n}^{2}\right)\right] q a & Q_{22}=\frac{1}{\sqrt{\chi}}|\boldsymbol{n}|^{2}\left[z M_{n}^{2}+\frac{\beta^{2}}{a^{2}}\left(1-M_{n}^{2}\right)\right] a^{2},
\end{array}
$$

with $z=0.5\left(1-\alpha+\frac{\beta^{2}}{a^{2}}\right)$ and

$$
\chi=(z q)^{2}+\frac{\beta^{2}}{a^{2}}\left(a^{2}|\boldsymbol{n}|^{2}-q^{2}\right)=a^{2}|\boldsymbol{n}|^{2}\left[z^{2} M_{n}^{2}+\frac{\beta^{2}}{a^{2}}\left(1-M_{n}^{2}\right)\right] .
$$

We also define

$$
\xi_{x}=\frac{\alpha u q-\beta^{2} n_{x}}{\left(\lambda_{+}-q\right)\left(\lambda_{-}-q\right)}, \quad \xi_{y}=\frac{\alpha v q-\beta^{2} n_{y}}{\left(\lambda_{+}-q\right)\left(\lambda_{-}-q\right)}
$$

and

$$
\zeta_{x}=\frac{n_{y}\left(u n_{y}-v n_{x}\right)}{\left(\lambda_{+}-q\right)\left(\lambda_{-}-q\right)}, \quad \zeta_{y}=-\frac{n_{x}\left(u n_{y}-v n_{x}\right)}{\left(\lambda_{+}-q\right)\left(\lambda_{-}-q\right)},
$$

with $\lambda_{ \pm}$given by (39). Using the identity $\left(\lambda_{+}-q\right)\left(\lambda_{-}-q\right)=\alpha q^{2}-\beta^{2}|\boldsymbol{n}|^{2}$, we have

$$
\xi_{x} n_{x}+\xi_{y} n_{y}=1
$$

We also see that

$$
\zeta_{x} n_{x}+\zeta_{y} n_{y}=0
$$

The rescaled dissipation matrix can be expressed as follows

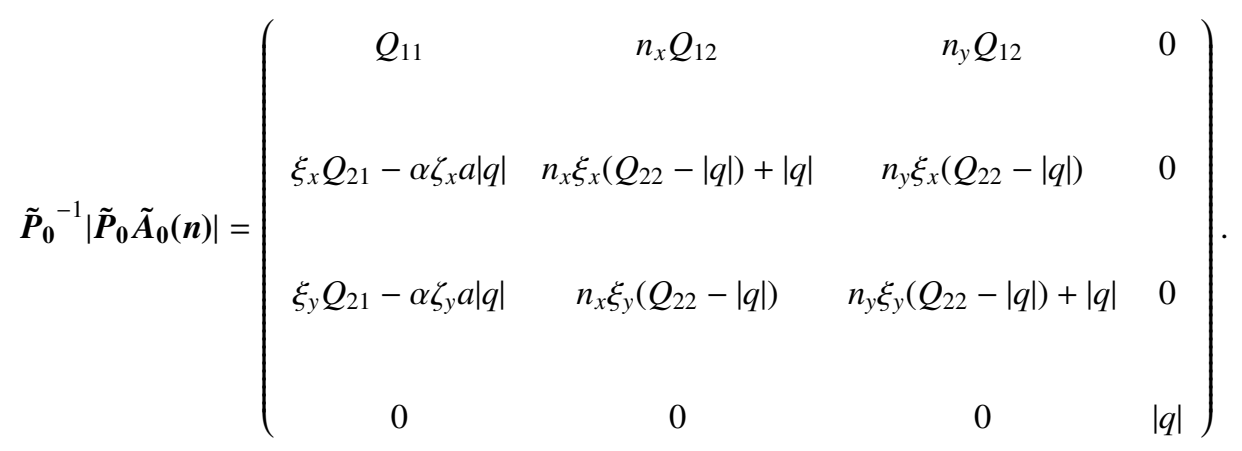

In the multidimensional case, we find the following decomposition

$$
\tilde{\boldsymbol{P}}_{\mathbf{0}}^{-1}\left|\tilde{\boldsymbol{P}}_{\mathbf{0}} \tilde{\boldsymbol{A}}_{\mathbf{0}}(\boldsymbol{n})\right|=\frac{1}{\sqrt{\chi}}\left[z q \tilde{\boldsymbol{A}}_{\mathbf{0}}(\boldsymbol{n})+\frac{\beta^{2}}{a^{2}}\left(a^{2}|\boldsymbol{n}|^{2}-q^{2}\right) \tilde{\boldsymbol{P}}_{\mathbf{0}}^{-1}\right]+\left(|q|-Q_{22}\right) \tilde{\boldsymbol{L}}_{\mathbf{0}}(\boldsymbol{n})
$$


with

$$
\tilde{\boldsymbol{L}}_{\mathbf{0}}(\boldsymbol{n})=\left(\begin{array}{cccc}
0 & 0 & 0 & 0 \\
-\alpha \zeta_{x} a & n_{y} \xi_{y} & 0 & 0 \\
-\alpha \zeta_{y} a & 0 & n_{x} \xi_{x} & 0 \\
0 & 0 & 0 & 1
\end{array}\right) .
$$

The matrix $\left(|q|-Q_{22}\right) \tilde{\boldsymbol{L}}_{\mathbf{0}}(\boldsymbol{n})$ has essentially $|q|-Q_{22}$ as non-zero eigenvalue with $|q| \simeq Q_{22}$ in the whole subsonic range except in the low speed limit. It doesn't affect the pressure equation. On the other hand, the resulting matrix of the two first contributions has the two acoustic eigenvalues $\mu_{ \pm}>0$ given in the following and double eigenvalue $Q_{22}>0$. So, this resulting matrix is close to the rescaled matrix dissipation (42). In particular, this "acoustic" contribution has the same behaviour as the rescaled matrix dissipation in the low speed limit. Additionally, when $\alpha=0$ we see that $\tilde{\boldsymbol{P}}_{\mathbf{0}}^{-1}$ and $\tilde{\boldsymbol{L}}_{\mathbf{0}}(\boldsymbol{n})$ have only diagonal entries and therefore the rescaled dissipation matrix is symmetric. At a sonic point, i.e. $|q|=a|\boldsymbol{n}|$, if $\alpha=0$ we also get $Q_{22}=a|\boldsymbol{n}|$. Therefore the two last contributions vanish and again for both possible values $q= \pm a|\boldsymbol{n}|$ we check that $\tilde{\boldsymbol{P}}_{\mathbf{0}}^{-1}\left|\tilde{\boldsymbol{P}}_{\mathbf{0}} \tilde{A}_{\mathbf{0}}(\boldsymbol{n})\right|=\left|\tilde{A}_{\mathbf{0}}(\boldsymbol{n})\right|$

In the incompressible limit, as $M \longrightarrow 0$, we have successively

$$
\chi \simeq|q|^{2}
$$

and for the coefficients $Q_{i j}$

$$
Q_{11} \simeq \frac{a^{2}}{|q|}|\boldsymbol{n}|^{2}=O\left(\frac{1}{M^{2}}\right), \quad Q_{12} \simeq a=O\left(\frac{1}{M}\right), \quad Q_{21} \simeq a|\boldsymbol{n}|^{2}=O\left(\frac{1}{M}\right), \quad Q_{22} \simeq|q|=O(1) .
$$

We also have

$$
\xi_{x} \simeq O(1), \quad \xi_{y} \simeq O(1), \quad \zeta_{x} \simeq O(1), \quad \zeta_{y} \simeq O(1) .
$$


Finally

$$
\tilde{\boldsymbol{P}}_{\mathbf{0}}^{-1}\left|\tilde{\boldsymbol{P}}_{\mathbf{0}} \tilde{\boldsymbol{A}}_{\mathbf{0}}(\boldsymbol{n})\right| \simeq\left(\begin{array}{cccc}
O\left(\frac{1}{M^{2}}\right) & O\left(\frac{1}{M}\right) & O\left(\frac{1}{M}\right) & 0 \\
O\left(\frac{1}{M}\right) & O(1) & O(1) & 0 \\
O\left(\frac{1}{M}\right) & O(1) & O(1) & 0 \\
0 & 0 & 0 & O(1)
\end{array}\right) \text { as } M \longrightarrow 0
$$

which gives by construction the proper scaling in the incompressible limit. Again, the rescaling can be interpreted as a net increase of numerical dissipation in the pressure equation by a factor $\frac{1}{M}$ and a consistent rescaling for the flow velocity.

From expression (42), we can easily find the eigenvalues $\mu$ of the matrix $\tilde{\boldsymbol{Q}}_{\mathbf{0}}$. Using the identities (40) and (41), we find for the linear waves

$$
\mu_{0}=|q| \quad \text { (double) }
$$

and for the acoustic waves

$$
\mu_{ \pm}=\frac{1}{2}\left[Q_{11}+Q_{22} \pm \sqrt{\left(Q_{11}-Q_{22}\right)^{2}+4 Q_{12} Q_{21}}\right] .
$$

Upon substitution with the expressions of the $Q_{i j}$

$$
\mu_{ \pm}=\frac{a|\boldsymbol{n}|}{2 \sqrt{\tilde{\chi}}}\left[2 z+\alpha\left(1-M_{n}^{2}\right) \pm \sqrt{\Delta}\right]
$$

$$
\text { with } \quad \Delta=\left(1-\frac{\beta^{2}}{a^{2}}\right)^{2}\left(1-M_{n}^{2}\right)^{2}+4 z\left[z+\alpha\left(1-M_{n}^{2}\right)\right] M_{n}^{2}, \quad \tilde{\chi}=z^{2} M_{n}^{2}+\frac{\beta^{2}}{a^{2}}\left(1-M_{n}^{2}\right) \text {. }
$$

We find as expected from the one-dimensional analysis that as long as the flow remains subsonic, the eigenvalues $\mu_{ \pm}$are positive with the following identity

$$
\mu_{+} \mu_{-}=a^{2}|\boldsymbol{n}|^{2}-q^{2}
$$

Thus, with $\alpha=0$ and $\mu_{0} \geq 0$, the rescaled dissipation matrix formulated for the set of independent variables $\boldsymbol{d} \tilde{\boldsymbol{W}}_{\mathbf{0}}$ is positive semidefinite in the subsonic range. For the two extreme cases

$$
\begin{aligned}
& \text { as } M_{n} \longrightarrow 0 \Rightarrow \mu_{+} \simeq \frac{a^{2}}{|\beta|}|\boldsymbol{n}|=O\left(\frac{1}{M^{2}}\right), \mu_{-} \simeq|\beta||\boldsymbol{n}|=O(1), \mu_{0} \simeq O(1) \\
& \text { as } M_{n} \longrightarrow 1 \Rightarrow \mu_{+} \simeq 2 a|\boldsymbol{n}|, \quad \mu_{-} \simeq 0, \quad \mu_{0} \simeq a|\boldsymbol{n}| .
\end{aligned}
$$


As anticipated in the one-dimensional case, we can see that severe stability constraints will occur in the incompressible limit, as it is discussed later.

In the multi-dimensional case, we derive the following diagonalization of the rescaled matrix dissipation

$$
\tilde{\boldsymbol{P}}_{\mathbf{0}}^{-1}\left|\tilde{\boldsymbol{P}}_{\mathbf{0}} \tilde{\boldsymbol{A}}_{\mathbf{0}}(\boldsymbol{n})\right|=\tilde{\boldsymbol{R}}_{\mathbf{0}} \tilde{\Lambda}_{\boldsymbol{R}}{ }^{-1}
$$

with $\tilde{\Lambda}=\operatorname{diag}\left(\mu_{+}, \mu_{-},|q|,|q|\right), \tilde{\boldsymbol{R}}_{\mathbf{0}}$ is the matrix of the right eigenvectors

$$
\tilde{\boldsymbol{R}}_{\mathbf{0}}=\left(\begin{array}{cccc}
\left(\mu_{+}-Q_{22}\right) & Q_{12} & 0 & 0 \\
{\left[\xi_{x} Q_{21}-\alpha \zeta_{x} a|q| R_{1}\right]} & {\left[\xi_{x}\left(\mu_{-}-Q_{11}\right)-\alpha \zeta_{x} a|q| R_{2}\right]} & -n_{y} & 0 \\
{\left[\xi_{y} Q_{21}-\alpha \zeta_{y} a|q| R_{1}\right]} & {\left[\xi_{y}\left(\mu_{-}-Q_{11}\right)-\alpha \zeta_{y} a|q| R_{2}\right]} & n_{x} & 0 \\
0 & 0 & 0 & 1
\end{array}\right)
$$

where

$$
R_{1}=\frac{\mu_{+}-Q_{22}}{\mu_{+}-|q|}, \quad R_{2}=\frac{Q_{12}}{\mu_{-}-|q|},
$$

and $\tilde{\boldsymbol{R}}_{\mathbf{0}}{ }^{-1}$ is the matrix of the left eigenvectors

$$
\tilde{\boldsymbol{R}}_{\mathbf{0}}^{-1}=\left(\begin{array}{cccc}
\frac{\left(\mu_{-}-Q_{11}\right)}{D} & -\frac{Q_{12}}{D} n_{x} & -\frac{Q_{12}}{D} n_{y} & 0 \\
-\frac{Q_{21}}{D} & \frac{\left(\mu_{+}-Q_{22}\right)}{D} n_{x} & \frac{\left(\mu_{+}-Q_{22}\right)}{D} n_{y} & 0 \\
-\alpha \zeta a|q| L_{1} & -\xi_{y}-\alpha \zeta_{y} a|q| L_{2} & \xi_{x}+\alpha \zeta_{x} a|q| L_{2} & 0 \\
0 & 0 & 0 & 1
\end{array}\right),
$$

with

$$
\zeta=\frac{\zeta_{x}}{n_{y}}=-\frac{\zeta_{y}}{n_{x}}=\frac{\left(u n_{y}-v n_{x}\right)}{\left(\lambda_{+}-q\right)\left(\lambda_{-}-q\right)}
$$

and

$$
L_{1}=\frac{Q_{22}-|q|}{\left(\mu_{+}-|q|\right)\left(\mu_{-}-|q|\right)}, \quad L_{2}=\frac{Q_{12}}{\left(\mu_{+}-|q|\right)\left(\mu_{-}-|q|\right)},
$$

where $D=\operatorname{det}\left(\tilde{\boldsymbol{R}}_{\mathbf{0}}\right)$ given by (33). 
The above scaling of the eigenvectors corresponds to the most straightforward extension of the one-dimensional formulation. It is worth noticing that the apparent singularity $\mu_{-}-|q|=0$ which may occur if $\alpha \neq 0$ for a specific directional Mach number $M_{n}<1$, root of the non-linear equation

$$
M_{n}^{2}+\frac{\mu_{+}}{a|n|} M_{n}-1=0
$$

doesn't create any numerical difficulty. We actually find by a deep inspection of the eigenspaces corresponding the second right eigenvector and the third left eigenvector that if $\mu_{-}=|q|$, then necessarily the velocity vector is aligned with the actual direction $\boldsymbol{n}$ and therefore $\zeta_{x}=\zeta_{y}=\zeta=0$. Thus, for this specific state, the second right eigenvector and the third left eigenvector recover non-singular expressions. It will be also shown in the stability analysis that $\mu_{+}>|q|$ for all possible values of the preconditioning parameter $\alpha$. So the above diagonalization can never become singular in practice in the subsonic range.

We also remind that by disactivating the preconditioning matrix with $\frac{\beta^{2}}{a^{2}}=1$ with $\alpha=0$, expressions (45)-(46) do not return the right and left eigenvector matrix of $\left|\tilde{A}_{\mathbf{0}}(\boldsymbol{n})\right|$, associated to eigenvalues $\left|\lambda_{+}\right|=a|\boldsymbol{n}|+q$ and $\left|\lambda_{-}\right|=a|\boldsymbol{n}|-q$. They actually correspond to the diagonalization of matrix $\left|\tilde{A}_{\mathbf{0}}(\boldsymbol{n})\right|$ with the acoustic eigenvalues recast for the module of the normal velocity $a|\boldsymbol{n}|+|q|$ and $a|\boldsymbol{n}|-|q|$. In the case $q \longrightarrow-a|\boldsymbol{n}|$, we especially see that $\left|\lambda_{+}\right| \longrightarrow 0$ and $\left|\lambda_{-}\right| \longrightarrow 2 a|\boldsymbol{n}|$ while $\mu_{+} \longrightarrow 2 a|\boldsymbol{n}|$ and $\mu_{-} \longrightarrow 0$ respectively. This justifies to formulate carefully a possible entropy fix at the sonic line as described later.

In the special case $\alpha=0$, we also see that the "shear" and "entropic" eigenvectors are both orthogonal to the acoustic eigenvectors. Taking $|\boldsymbol{n}|=1$, with (34) the acoustic eigenvectors are also orthogonal to each others and the diagonalization with unitary congruence (35)-(36) can be applied. So the symmetry properties of the original unpreconditioned system are preserved for the rescaled matrix dissipation. However, this is no longer true when the rescaled matrix dissipation is formulated from the primitive or conservative variables since the eigenvectors are modified by any change of variables. We know from [21] that the non normality of the preconditioned system leads to significant robustness problems at stagnation points.

The formulation of the rescaled matrix dissipation expressed for the conservative variables $\boldsymbol{W}$ is achieved by additional similarity transformations, using the intermediate primitive variables $\boldsymbol{W}_{\mathbf{0}}=[p, u, v, S]^{T}$ and applying the change of variables with the similarity matrix $\frac{\partial \boldsymbol{W}}{\partial \tilde{\boldsymbol{W}}_{\mathbf{0}}}=$ 
$\frac{\partial \boldsymbol{W}}{\partial \boldsymbol{W}_{\mathbf{0}}} \frac{\partial \boldsymbol{W}_{\mathbf{0}}}{\partial \tilde{\boldsymbol{W}}_{\mathbf{0}}}$

\section{Stability analysis for the explicit scheme}

We now consider the explicit scheme with rescaling of the dissipation matrix derived in the previous section, expressed for the conservative variables $\boldsymbol{W}$

$$
\frac{\partial \boldsymbol{W}}{\partial t}=-\left[\frac{\partial \boldsymbol{F}}{\partial x}+\frac{\partial \boldsymbol{G}}{\partial y}-\frac{\partial}{\partial x}\left(\boldsymbol{Q}_{x} \frac{\partial \boldsymbol{W}}{\partial x}\right)-\frac{\partial}{\partial y}\left(\boldsymbol{Q}_{y} \frac{\partial \boldsymbol{W}}{\partial y}\right)\right] .
$$

In the two-dimensional case, the explicit time-discretization at mesh point $\boldsymbol{x}_{i j}=(i \delta x, j \delta y)$, where $\delta x$ and $\delta y$ are the constant mesh spacing in the $(x, y)$ directions, can be formulated in conservation form

$$
\frac{\Delta \boldsymbol{W}_{i j}}{\Delta t}+\frac{\boldsymbol{h}_{i+\frac{1}{2} j}-\boldsymbol{h}_{i-\frac{1}{2} j}}{\delta x}+\frac{\boldsymbol{g}_{i j+\frac{1}{2}}-\boldsymbol{g}_{i j-\frac{1}{2}}}{\delta y}=0,
$$

with the time increment $\Delta \boldsymbol{W}_{i j}=\boldsymbol{W}_{i j}^{n+1}-\boldsymbol{W}_{i j}^{n}$. For the first-order Roe scheme, the numerical flux writes in the $\mathrm{x}$-direction

$$
\boldsymbol{h}_{i+\frac{1}{2} j}=\frac{1}{2}\left[\boldsymbol{F}\left(\boldsymbol{W}_{i j}^{n}\right)+\boldsymbol{F}\left(\boldsymbol{W}_{i+1 j}^{n}\right)\right]-\frac{1}{2}\left|\boldsymbol{A}_{\text {roe }}\right|_{i+\frac{1}{2} j}\left(\boldsymbol{W}_{i+1 j}^{n}-\boldsymbol{W}_{i j}^{n}\right) .
$$

For the rescaled Roe scheme, we substitute (48) with

$$
\boldsymbol{h}_{i+\frac{1}{2} j}=\frac{1}{2}\left[\boldsymbol{F}\left(\boldsymbol{W}_{i j}^{n}\right)+\boldsymbol{F}\left(\boldsymbol{W}_{i+1 j}^{n}\right)\right]-\frac{1}{2} \boldsymbol{P}^{-1}|\boldsymbol{P A}|_{i+\frac{1}{2} j}\left(\boldsymbol{W}_{i+1 j}^{n}-\boldsymbol{W}_{i j}^{n}\right),
$$

with matrix $\boldsymbol{P}^{-1}|\boldsymbol{P A}|$ formulated for the Roe average. There is no need to explicitly formulate the matrix dissipation for the conservative variables. In practice, the dissipation vector can always be computed using similarity transformations following the algebraic algorithm described in [15] for the iterative preconditioning

$$
\boldsymbol{P}^{-1}|\boldsymbol{P A}|_{i+\frac{1}{2} j}\left(\boldsymbol{W}_{i+1 j}^{n}-\boldsymbol{W}_{i j}^{n}\right)=\left[\frac{\partial \boldsymbol{W}}{\partial \tilde{\boldsymbol{W}}_{\mathbf{0}}} \tilde{\boldsymbol{R}}_{\mathbf{0}} \tilde{\boldsymbol{\Lambda}} \tilde{\boldsymbol{R}}_{\mathbf{0}}^{-1} \frac{\partial \tilde{\boldsymbol{W}}_{\mathbf{0}}}{\partial \boldsymbol{W}}\right]_{i+\frac{1}{2} j}\left(\boldsymbol{W}_{i+1 j}-\boldsymbol{W}_{i j}\right) .
$$

The stability constraint is strongly concerned with the spectral radius $\rho\left(\boldsymbol{P}^{-1}|\boldsymbol{P A}|\right)$ of the matrixvalued dissipation. We have $\rho\left(\boldsymbol{P}^{-\mathbf{1}}|\boldsymbol{P} \boldsymbol{A}|\right)=\max \left(\mu_{+},|q|\right)$ since $\mu_{+}>\mu_{-}$. We found that $\mu_{+}>|q|$ when $M_{n} \rightarrow 0$ and $M_{n} \rightarrow 1$. This is actually true $\forall M_{n} \leq 1$ and for $\alpha=0, \alpha=1$ and $\alpha=-1$ as demonstrated in the following.

Using identity (44) and taking advantage of having both $\mu_{ \pm} \geq 0$, we can derive the following expression

$$
\left(\mu_{+}-|q|\right)\left(\mu_{-}+|q|\right)=a_{25}^{2}|\boldsymbol{n}|^{2}\left[1+M_{n} \sqrt{\frac{\Delta}{\tilde{\chi}}}-2 M_{n}^{2}\right] .
$$


From (50), we have the sufficient condition that as long as $M_{n} \leq \frac{\sqrt{2}}{2}$, then $\mu_{+}-|q| \geq 0$ and $\rho\left(\boldsymbol{P}^{-1}|\boldsymbol{P A}|\right)=\mu_{+}$, without condition on the free parameter $\alpha$. We also find that if $\Delta-\tilde{\chi} \geq 0$, then $\sqrt{\frac{4}{\tilde{\chi}}} \geq 1$ and $\mu_{+}-|q| \geq 0$ with

$$
1+M_{n} \sqrt{\frac{\Delta}{\tilde{\chi}}}-2 M_{n}^{2} \geq 1+M_{n}-2 M_{n}^{2} \geq 0 \text { for all } \quad 0 \leq M_{n} \leq 1 .
$$

We can formulate the quantity $\Delta-\tilde{\chi}$ as follows

$$
\Delta-\tilde{\chi}=P_{0}\left(1-M_{n}^{2}\right)+P_{1} M_{n}^{2}
$$

with

$$
P_{0}=\left(1-\frac{\beta^{2}}{a^{2}}\right)^{2}\left(1-M_{n}^{2}\right)+2 \alpha\left(1-\alpha+\frac{\beta^{2}}{a^{2}}\right) M_{n}^{2}-\frac{\beta^{2}}{a^{2}} \quad \text { and } \quad P_{1}=\frac{3}{4}\left(1-\alpha+\frac{\beta^{2}}{a^{2}}\right)^{2}
$$

Since $P_{1}>0$, a sufficient condition for $\Delta-\tilde{\chi} \geq 0$ is $P_{0} \geq 0$. Taking $\alpha=1$, we see that as long as $M_{n}$ lies in the range $\frac{\sqrt{2}}{2} \leq M_{n} \leq 1, P_{0} \geq 0$. Thus setting $\alpha=1$ ensures $\mu_{+}-|q|>0$ and then $\rho\left(\boldsymbol{P}^{-1}|\boldsymbol{P} \boldsymbol{A}|\right)=\mu_{+}$for all $M_{n} \leq 1$. On the other hand, considering now the cases $\alpha=0$ and $\alpha=-1$, we cannot conclude on the sign of $\mu_{+}-|q|$ by using the expression $\Delta-\tilde{\chi}$. However, using identity (50), we see that

$$
\left(\mu_{+}-|q|\right)\left(\mu_{-}+|q|\right)\left(1+M_{n} \sqrt{\frac{\Delta}{\tilde{\chi}}}+2 M_{n}^{2}\right)=a^{2}|\boldsymbol{n}|^{2}\left[1+2 M_{n} \sqrt{\frac{\Delta}{\tilde{\chi}}}+M_{n}^{2}\left(\frac{\Delta-4 M_{n}^{2} \tilde{\chi}}{\tilde{\chi}}\right)\right],
$$

with $\tilde{\chi}>0$. Following the same idea, we also have

$$
\left(\mu_{+}-\rho(\boldsymbol{A})\right)\left(\mu_{-}+\rho(\boldsymbol{A})\right)\left(\sqrt{\frac{\Delta}{\tilde{\chi}}}+2 M_{n}\right)=a^{2}|\boldsymbol{n}|^{2}\left(1+M_{n}\right)\left(\frac{\Delta-4 M_{n}^{2} \tilde{\chi}}{\tilde{\chi}}\right),
$$

where $\rho(\boldsymbol{A})=|q|+a|\boldsymbol{n}|$ is the spectral radius for the unpreconditioned scheme.

With $\alpha=0$

$$
\Delta-4 M_{n}^{2} \tilde{\chi}=\left(1-\frac{\beta^{2}}{a^{2}}\right)^{2}\left(1-M_{n}^{4}\right) \geq 0 \quad \text { for all } \quad 0 \leq M_{n} \leq 1 .
$$

With $\alpha=-1$

$$
\Delta-4 M_{n}^{2} \tilde{\chi}=\left(\left(1-\frac{\beta^{2}}{a^{2}}\right)^{2}-M_{n}^{2}\right)\left(1-M_{n}^{2}\right) \quad \Rightarrow \quad 1+M_{n}^{2}\left(\frac{\Delta-4 M_{n}^{2} \tilde{\chi}}{\tilde{\chi}}\right)>0 .
$$

We then conclude that

$$
\rho\left(\boldsymbol{P}^{-1}|\boldsymbol{P A}|\right)=\mu_{+} \quad \forall M_{n} \leq 1,
$$


for all possible values of the parameter $\alpha$. Furthermore, if $\alpha=0$, we also have $\mu_{+} \geq \rho(\boldsymbol{A})$ for all $M_{n} \leq 1$ which is not always the case with $\alpha=1$ or $\alpha=-1$.

In the framework of a linear Von-Neumann analysis, $\boldsymbol{W}_{i j}^{n}$ is replaced by its Fourier expansion. The Jacobian matrices $\boldsymbol{A}$ and $\boldsymbol{P}^{-1}|\boldsymbol{P A}|$ are assumed constant matrices and the amplification matrix corresponding to the explicit scheme (47) with the numerical flux (49) is given for one space-dimension by

$$
\boldsymbol{G}(\phi, \sigma)=\boldsymbol{I} \boldsymbol{d}+(\cos (\phi)-1) \sigma \boldsymbol{P}^{-\mathbf{1}}|\boldsymbol{P A}|-i \sin (\phi) \sigma \boldsymbol{A},
$$

where $\phi$ is the wave number (normalized by $1 / \delta x$ ), $\sigma=\frac{\Delta t}{\delta x}$ and $i^{2}=-1$. This amplification matrix is the same as for the preconditioned Lax-Friedrich type schemes studied in [9]. Let $\epsilon>0$. Then, there exits some subordinate matrix-norm $\|.\|_{G, \epsilon}$ depending on $\boldsymbol{G}$ and $\epsilon$ such that

$$
\rho(\boldsymbol{G}) \geq\left\|\boldsymbol{I} \boldsymbol{d}+(\cos (\phi)-1) \sigma \boldsymbol{P}^{-1}|\boldsymbol{P A}|-i \sin (\phi) \sigma \boldsymbol{A}\right\|_{G, \epsilon}-\epsilon .
$$

We also have for any matrix-norm

$$
\|\boldsymbol{A}\| \geq \rho(\boldsymbol{A}) \simeq O\left(\frac{1}{M}\right) \text { and }\left\|\boldsymbol{P}^{-1} \mid \boldsymbol{P} \boldsymbol{A}\right\| \| \geq \rho\left(\boldsymbol{P}^{-\mathbf{1}}|\boldsymbol{P} \boldsymbol{A}|\right) \simeq O\left(\frac{1}{M^{2}}\right)>>\rho(\boldsymbol{A}) \text { as } M \rightarrow 0 .
$$

Therefore, the Von-Neumann stability condition gives a stringent constraint on the time-step in the incompressible limit as formulated in [9], with for a fixed $\delta x$

$$
1 \geq \rho(\boldsymbol{G}) \geq\|\boldsymbol{G}\|_{G, \epsilon}-\epsilon \geq \frac{\Delta t}{\delta x} \rho\left(\boldsymbol{P}^{-1}|\boldsymbol{P} \boldsymbol{A}|\right)-\epsilon \simeq O\left(\frac{\Delta t}{M^{2}}\right) \Longrightarrow \Delta t \simeq O\left(M^{2}\right) \text { as } M \rightarrow 0 .
$$

This proof can be extended to the multi-dimensional formulation in a straightforward way. From (51), we conclude that the stability condition for the explicit rescaled Roe scheme is formulated for the fasted wave speed as follows

$$
\Delta t \leq \frac{h}{\rho\left(\boldsymbol{P}^{-1}|\boldsymbol{P} \boldsymbol{A}|\right)},
$$

where $h$ represents some characteristic cell distance. In the two extreme cases, the stability condition yields

$$
\Delta t \leq \frac{h}{\mu_{+}} \simeq O\left(M^{2}\right) \text { as } M \rightarrow 0,
$$

and

$$
\Delta t \leq \frac{h}{|q|+a|\boldsymbol{n}|} \text { as } M \rightarrow 1
$$


So for steady-state problems, the local time step will be very small $\simeq O\left(h M^{2}\right)$ in the lowspeed flow regions only. In supersonic flow regions, the "standard" stability condition (53) must be applied. Therefore, with the strong limitation on the time step, an efficient implicit stage must be added to the baseline explicit rescaled Roe scheme as described in the next section for the steady-state problem.

It is interesting to compare the acoustic eigenvalues $\mu_{ \pm}$of the rescaled dissipation matrix and the original eigenvalues $\left|\lambda_{ \pm}\right|$in the whole subsonic range $M_{n} \leq 1$. In the next figures, all eigenvalues are normalized by the local speed of sound and therefore can be expressed only as functions of the dimensionless normal velocity $\frac{q}{a|\boldsymbol{n}|} \in[-1,1]$ (= $\frac{u}{a}$ in the one-dimensional case). The eigenvalues $\mu_{ \pm}$strongly depend on the local Mach number $M$ within the parameter $\frac{\beta^{2}}{a^{2}}$ with a noticeable effect of the second parameter $\alpha$ for low Mach numbers. In Fig. 1-3 we have compared the effect of $\alpha=-1, \alpha=0$ and $\alpha=1$, considering different values of the local Mach number. As demonstrated above, we see that $\mu_{+}$is the largest eigenvalue in the entire subsonic range with $\mu_{+}>|q|, \forall \alpha, \mu_{+} \geq \rho(\boldsymbol{A})$ for $\alpha=0$ and that $\mu_{-}$takes small values especially when $M \leq 0.1$. The value of $\alpha$ has little impact on $\mu_{-}$but a large effect on $\mu_{+}$in the incompressible limit, as illustrated in Fig. 1 for $M=0.1$. As it can be clearly seen, the stiffness of the rescaled dissipation matrix increases dramatically as the local Mach number is becoming smaller and smaller. The worst situation arises for $\alpha=1$, which could not be tested in our computations of low Mach-number flows. Globally $\alpha=-1$ gives the better conditioning except when $\frac{q}{a|\boldsymbol{n}|} \rightarrow 0$ where the asymptotic value of $\mu_{+}$is reached. This asymptotic value given by $\frac{a^{2}}{|\beta|}|\boldsymbol{n}|$ is independent of $\alpha$ but strongly depends on the local Mach number. When $M \rightarrow 1$, the effect of $\alpha$ is almost negligible and the stiffness of the rescaled Roe matrix becomes identical to the original Roe matrix (see Fig. 3 for the case $M=1$ with $\mu_{+}=\rho(\boldsymbol{A})$ if $\alpha=0$ ). 


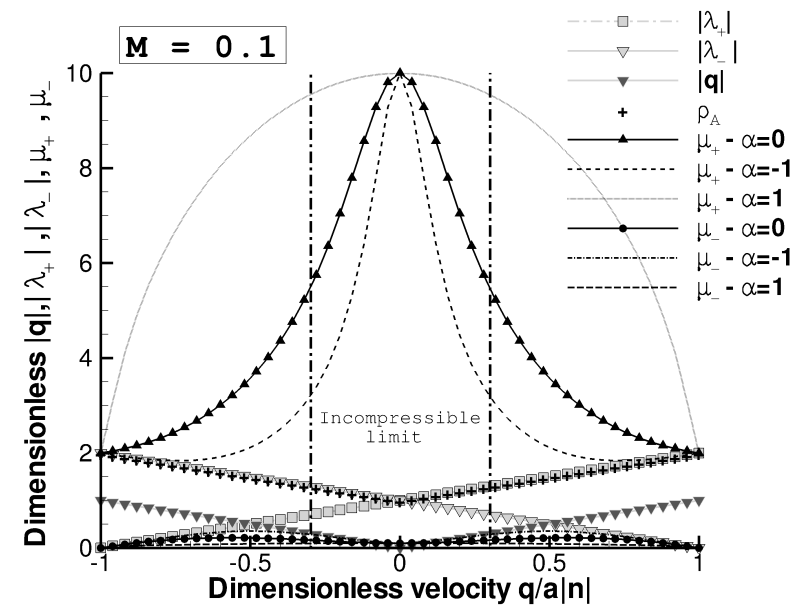

Figure 1: Dimensionless eigenvalues for the original matrix-valued dissipation (Roe) and for the rescaled Roe scheme when $M=0.1$

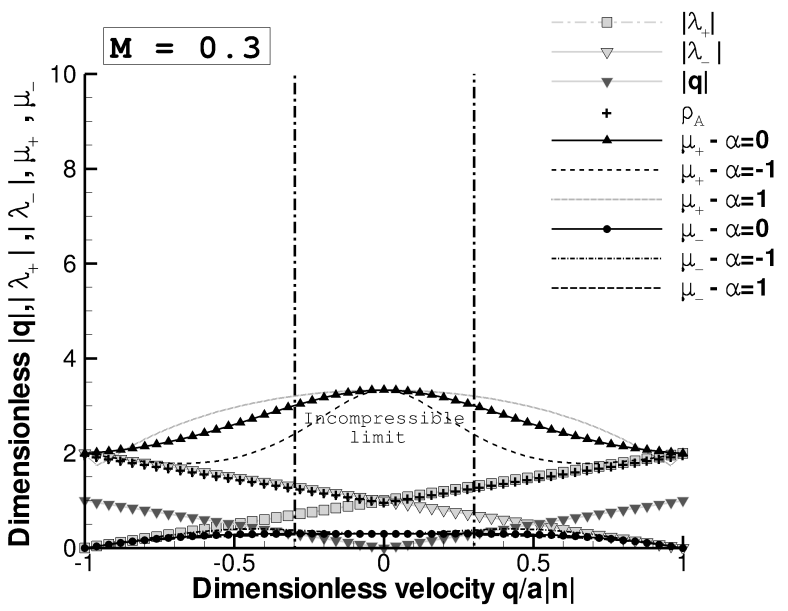

Figure 2: Dimensionless eigenvalues for the original matrix-valued dissipation (Roe) and for the rescaled Roe scheme when $M=0.3$

\section{Implicit matrix time-stepping scheme for the steady-state problem}

\subsection{Formulation}

The Jacobi preconditioning or preconditioning-squared is known to smooth out high frequency errors and is especially efficient with the multigrid technique to accelerate the convergence. It basically doesn't help to improve the stiffness of the rescaled Roe scheme in the low 


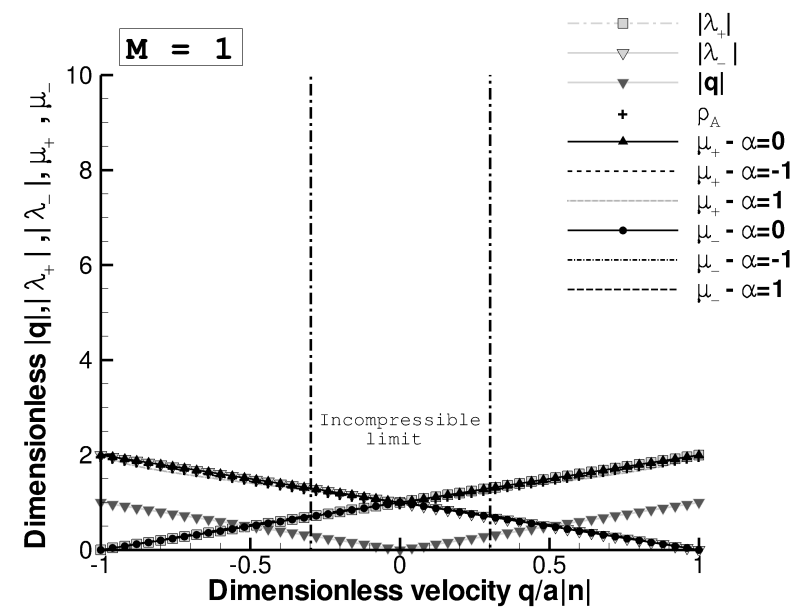

Figure 3: Dimensionless eigenvalues for the original matrix-valued dissipation (Roe) and for the rescaled Roe scheme when $M=1$

speed limit. On the other hand the low-speed preconditioning is not designed to treat high frequency errors. Turkel suggested to combine both approaches. However, here we apply a block Jacobi preconditioner to the rescaled Roe scheme in order to formulate an implicit matrix timestepping scheme for the steady-state problem in the sens that each characteristic variables is updated with its own $\Delta t^{(k)}$ [22]. Actually the matrix time-stepping approach provides a mechanism enforcing the damping properties of the scheme as it is described next within a linear stability analysis. In the case of the rescaled Roe scheme, the formulation of a time-step matrix is not obvious, since the dissipation matrix is not an explicit function of the original Jacobian matrix. For the fully discrete scheme in a two-dimensional Cartesian mesh (47) with the numerical flux corresponding to the rescaled Roe scheme (49), the Jacobi preconditioning may be formulated as follows

$$
\left(\frac{\boldsymbol{A}_{\boldsymbol{p}}+\boldsymbol{B}_{\boldsymbol{p}}}{\rho\left(\boldsymbol{P}^{-1}|\boldsymbol{P A}|\right)+\rho\left(\boldsymbol{P}^{-1}|\boldsymbol{P B}|\right)} \Delta \boldsymbol{W}\right)_{i j}=-\Delta t\left(\frac{\boldsymbol{h}_{i+\frac{1}{2} j}-\boldsymbol{h}_{i-\frac{1}{2} j}}{\delta x}+\frac{\boldsymbol{g}_{i j+\frac{1}{2}}-\boldsymbol{g}_{i j-\frac{1}{2}}}{\delta y}\right),
$$

where the matrix coefficients $\boldsymbol{A}_{\boldsymbol{p}}$ and $\boldsymbol{B}_{\boldsymbol{p}}$ must fulfill the following conditions:

1. The eigenvectors must be the same as the original Jacobian matrix in order to preserve the formulation of the characteristics variables;

2. The eigenvalues must be non negative in order to let unchanged the signs of the characteristic speeds and bounded away from zero (thus positive) for the formulation of a characteristic time-step matrix; 
3. The stability condition (52) must be satisfied for the characteristic variable corresponding to the fastest wave speed;

4. The matrix coefficients $\boldsymbol{A}_{\boldsymbol{p}}$ and $\boldsymbol{B}_{\boldsymbol{p}}$ should achieve a smooth transition with $|\boldsymbol{A}|$ and $|\boldsymbol{B}|$ at the sonic line.

Matrix coefficients $\boldsymbol{A}_{p}=\boldsymbol{P}^{-1}|\boldsymbol{P A}|$ and $\boldsymbol{B}_{p}=\boldsymbol{P}^{-1}|\boldsymbol{P} \boldsymbol{B}|$ perfectly match conditions 2., 3. and 4. but must be rejected as not satisfying the first condition. Setting $\boldsymbol{A}_{p}=|\boldsymbol{A}|=\boldsymbol{R}_{\boldsymbol{A}}\left|\boldsymbol{\Lambda}_{\boldsymbol{A}}\right| \boldsymbol{R}_{\boldsymbol{A}}{ }^{-1}$ and $\boldsymbol{B}_{p}=|\boldsymbol{B}|=\boldsymbol{R}_{\boldsymbol{B}}\left|\boldsymbol{\Lambda}_{\boldsymbol{B}}\right| \boldsymbol{R}_{\boldsymbol{B}}{ }^{-1}$ satisfy conditions 1., 2. and 4., provided that the vanishing eigenvalues $\left|\lambda^{(k)}\right|$ are corrected by some entropy fix. This latter choice reduces the spread of the characteristic speeds from a factor $1 / M$ with respect to the formulation with the rescaled dissipation matrix. However, condition 3. is lost with $\rho(\boldsymbol{A}) \leq \mu_{+}$and in practice, this formulation is unstable and can be used only with small or moderate values of the CFL number. A intermediate choice is to build matrix coefficients from the eigenvectors of the original Jacobian matrix and the eigenvalues $\mu^{(k)}$ of the rescaled matrix dissipation supposed to be all bounded away from zero. In that case, the eigenvalues $\mu^{(k)}$ must be corrected to ensure the transition at the sonic line with $\left|\lambda^{(k)}\right|$ the eigenvalues of the original Roe matrix dissipation. This intermediate formulation provides optimal stability properties and yields a robust scheme but let the spread of the characteristic speeds unchanged with respect to the rescaled Roe scheme.

Hence, let consider $\boldsymbol{A}_{p}=\boldsymbol{R}_{A} \tilde{\boldsymbol{\Lambda}}_{A} \boldsymbol{R}_{A}^{-1}$ and $\boldsymbol{B}_{p}=\boldsymbol{R}_{\boldsymbol{B}} \tilde{\boldsymbol{\Lambda}}_{\boldsymbol{B}} \boldsymbol{R}_{\boldsymbol{B}}{ }^{-1}$, with $\tilde{\boldsymbol{\Lambda}}=\operatorname{diag}\left(\mu^{(k)}\right), \boldsymbol{R}_{A}$ and $\boldsymbol{R}_{\boldsymbol{B}}$ being the left eigenvector matrices of the original Jacobian matrices $\boldsymbol{A}$ and $\boldsymbol{B}$. In a Cartesian mesh, the CFL condition (52) can be expressed with a more restrictive formulation of the local time-step

$$
\Delta t=C F L \frac{h}{\rho\left(\boldsymbol{P}^{-1}|\boldsymbol{P A}|\right)+\rho\left(\boldsymbol{P}^{-1}|\boldsymbol{P} \boldsymbol{B}|\right)} .
$$

We introduce a characteristic time-step matrix

$$
\Delta t_{c}=C F L h\left(A_{p}+B_{p}\right)^{-1}=\left[\left(\boldsymbol{R}_{A} \Delta t_{A}^{-1} \boldsymbol{R}_{A}^{-1}\right)+\left(\boldsymbol{R}_{B} \Delta t_{B}{ }^{-1} \boldsymbol{R}_{B}^{-1}\right)\right]^{-1},
$$

where $\Delta t$ is the diagonal matrix

$$
\Delta \boldsymbol{t}=\operatorname{diag}\left(\Delta t^{+}, \Delta t^{-}, \Delta t^{0}, \Delta t^{0}\right)
$$

with

$$
\Delta t^{+}=C F L \frac{h}{\mu_{*}^{+}}, \quad \Delta t^{-}=C F L \frac{h}{\mu_{*}^{-}}, \quad \Delta t^{0}=C F L \frac{h}{\mu_{*}^{0}} .
$$


The star symbol indicates that the eigenvalues may be bounded away from zero and especially that $\mu_{ \pm}$must be modified to ensure that the above condition 4 is satisfied. This point is discussed later, in the supersonic flow extension of the rescaled Roe scheme.

Thus, the Jacobi preconditioner amounts to reformulate the original conservative scheme (47) with a time-step matrix

$$
\Delta \boldsymbol{W}_{i j}=-\left(\Delta \boldsymbol{t}_{\boldsymbol{c}}\right)_{i j}\left(\frac{\boldsymbol{h}_{i+\frac{1}{2} j}-\boldsymbol{h}_{i-\frac{1}{2} j}}{\delta x}+\frac{\boldsymbol{g}_{i j+\frac{1}{2}}-\boldsymbol{g}_{i j-\frac{1}{2}}}{\delta y}\right) .
$$

This scheme has optimal properties in damping high frequency errors, and is well adapted to transonic flows. By setting $\boldsymbol{P}$ to the identity matrix $\boldsymbol{I d}$ in (54), we can also formulate a matrix time-stepping scheme for the original Roe scheme with

$$
\Delta t^{+}=C F L \frac{h}{\left|\lambda_{+}\right|}, \quad \Delta t^{-}=C F L \frac{h}{\left|\lambda_{-}\right|}, \quad \Delta t^{0}=C F L \frac{h}{\left|\lambda_{0}\right|} .
$$

and $\lambda_{ \pm}=q \pm a|\boldsymbol{n}|, \lambda_{0}=q$. Note that if $\Delta t^{-}$and $\Delta t^{0}$ are set to the maximum value allowed by the CFL condition, we recover the usual local time step with $\Delta \boldsymbol{t}_{c}=\Delta t \boldsymbol{I d}$. In the matrix timestep approach, we would like to let the time steps associated to the slowest waves unbounded. Intuitively, since in the incompressible limit we have for a fixed mesh

$$
\Delta t^{+} \simeq h C F L O\left(M^{2}\right), \quad \Delta t^{-} \simeq h C F L O(1), \quad \Delta t^{0} \simeq h C F L O(1),
$$

the original idea behind the formulation of a time-step matrix was to modify the characteristic time-steps so that the solution advance in time along the characteristic curves with time steps of the same order of magnitude. This is actually not suitable, since it is essential to keep the numerical stability of the rescaled Roe scheme. Equalizing all characteristic time steps would give the additional constraint that $\Delta t^{-}$and $\Delta t^{0}$ must be scaled to the smallest time step $\Delta t^{+}$, and we recover the behaviour of the numerical scheme formulated for the scalar time step. In order to overcome the issue of using very small time steps in the incompressible limit, we see that the matrix time-stepping scheme must be combined with a robust implicit scheme allowing the use of large CFL numbers, as recommended in [9].

A usual way to define an implicit scheme for the Roe scheme, is to consider the following backward Euler scheme as formulated in [23] for an approximated differentiation of the Roe flux

$$
\begin{array}{ll}
-\sigma_{x} \boldsymbol{A}^{+}{ }_{i-\frac{1}{2} j} \Delta \boldsymbol{W}_{i-1 j}+\sigma_{x} \boldsymbol{A}^{-}{ }_{i+\frac{1}{2} j} \Delta \boldsymbol{W}_{i+1 j} & \\
+\left(\boldsymbol{I} \boldsymbol{d}-\sigma_{x} \boldsymbol{A}^{-}{ }_{i+\frac{1}{2} j}+\sigma_{x} \boldsymbol{A}^{+}{ }_{i-\frac{1}{2} j}-\sigma_{y} \boldsymbol{B}^{-}{ }_{i j+\frac{1}{2}}+\sigma_{y} \boldsymbol{B}^{+}{ }_{i j-\frac{1}{2}}\right) \Delta \boldsymbol{W}_{i j} & \\
-\sigma_{y} \boldsymbol{B}^{+}{ }_{i j-\frac{1}{2}} \Delta \boldsymbol{W}_{i j-1}+\sigma_{y} \boldsymbol{B}^{-}{ }_{i j+\frac{1}{2}} \Delta \boldsymbol{W}_{i j+1} & =\Delta \boldsymbol{W}_{i j}^{e x p}
\end{array}
$$


where $\Delta \boldsymbol{W}_{i j}^{e x p}$ is the time increment for the explicit scheme, $\sigma_{x}=\frac{\Delta t}{\delta x}, \sigma_{y}=\frac{\Delta t}{\delta y}$ and $\boldsymbol{A}^{+}, \boldsymbol{A}^{-}$, $\boldsymbol{B}^{+}, \boldsymbol{B}^{-}$are respectively the positive and negative parts of the flux Jacobian matrices $\boldsymbol{A}$ and $\boldsymbol{B}$ in the $(x, y)$ space directions. For the rescaled Roe scheme, a baseline implicit scheme is then formulated by replacing in (55) respectively

$$
\boldsymbol{A}^{ \pm} \longmapsto \frac{\boldsymbol{A} \pm \boldsymbol{P}^{-1}|\boldsymbol{P A}|}{2}=\boldsymbol{P}^{-1}(\boldsymbol{P A})^{ \pm} \quad \text { and } \quad \boldsymbol{B}^{ \pm} \longmapsto \frac{\boldsymbol{B} \pm \boldsymbol{P}^{-1}|\boldsymbol{P} \boldsymbol{B}|}{2}=\boldsymbol{P}^{-1}(\boldsymbol{P} \boldsymbol{B})^{ \pm} .
$$

The implicit scheme (55) can be also formulated for the characteristic matrix time-stepping scheme, upon the following substitutions

$$
\sigma_{x} \boldsymbol{I} \boldsymbol{d} \longmapsto \frac{1}{\delta x} \boldsymbol{\Delta} \boldsymbol{t}_{c}, \quad \sigma_{y} \boldsymbol{I} \boldsymbol{d} \longmapsto \frac{1}{\delta y} \Delta \boldsymbol{t}_{c} .
$$

\subsection{Stability of the implicit scheme}

In the following, the stability of the implicit rescaled Roe scheme with scalar and matrix time-steps is considered. Both versions correspond actually to small perturbations of the original implicit Roe scheme (55) in the transonic regime. Both are especially unconditionally stable for the linear problem as shown below. But as the free-stream Mach number is decreasing, their dissipative properties substantially differ from the original Roe scheme. This can be pointed out within a Fourier analysis. Let $(\phi, \theta)$ be the normalized wave numbers in the two space directions. Then, for the linear problem the amplification matrix takes the general form

$$
\boldsymbol{G}(\phi, \theta)=\boldsymbol{I} \boldsymbol{d}-\boldsymbol{H}^{-1}(\phi, \theta) \boldsymbol{K}(\phi, \theta),
$$

with for the implicit scheme $\boldsymbol{H}(\phi, \theta)=\boldsymbol{I} \boldsymbol{d}+\boldsymbol{K}(\phi, \theta)$. We get successively

- For the original Roe scheme

$$
\boldsymbol{K}(\phi, \theta)=(1-\cos (\phi)) \sigma_{x}|\boldsymbol{A}|+(1-\cos (\theta)) \sigma_{y}|\boldsymbol{B}|+i \sin (\phi) \sigma_{x} \boldsymbol{A}+i \sin (\theta) \sigma_{y} \boldsymbol{B}
$$

- For the rescaled Roe scheme

$$
\boldsymbol{K}(\phi, \theta)=(1-\cos (\phi)) \sigma_{x} \boldsymbol{P}^{-1}|\boldsymbol{P A}|+(1-\cos (\theta)) \sigma_{y} \boldsymbol{P}^{-1}|\boldsymbol{P} \boldsymbol{B}|+i \sin (\phi) \sigma_{x} \boldsymbol{A}+i \sin (\theta) \sigma_{y} \boldsymbol{B}
$$

- For the the matrix time-stepping schemes, we replace $\sigma_{x}$ and $\sigma_{y}$ according to (57).

Stability and damping properties of the four schemes can be characterized by the following estimates, assuming that all matrix coefficients simultaneously diagonalizable according to the Lerat's general framework [24]. Let introduce $\lambda_{\boldsymbol{G}}^{(k)}$ the eigenvalues of the amplification matrix 
$\boldsymbol{G}(\phi, \theta)$ and respectively $\lambda_{\boldsymbol{A}}^{(k)}, \lambda_{\boldsymbol{B}}^{(k)}$ and $\mu_{\boldsymbol{A}}^{(k)}, \mu_{\boldsymbol{B}}^{(k)}$ the eigenvalues of the matrix coefficients $\boldsymbol{A}, \boldsymbol{B}$ and $\boldsymbol{A}_{p}, \boldsymbol{B}_{p}, \boldsymbol{P}^{-1}|\boldsymbol{P} \boldsymbol{A}|$ and $\boldsymbol{P}^{-1}|\boldsymbol{P} \boldsymbol{B}|$. Under this assumption, the time-step matrix has eigenvalues

$$
\Delta t^{(k)}=C F L \frac{h}{\left|\lambda_{\boldsymbol{A}}^{(k)}\right|+\left|\lambda_{\boldsymbol{B}}^{(k)}\right|} \quad \text { (Roe scheme), } \Delta t^{(k)}=C F L \frac{h}{\mu_{\boldsymbol{A}}^{(k)}+\mu_{\boldsymbol{B}}^{(k)}} \quad \text { (rescaled Roe scheme). }
$$

Important for differentiating the different formulations is to have a close upper estimate of the spectral radius of the amplification matrix. Without loss of generality, we have for the Roe scheme in one space-dimension

$$
\rho(\boldsymbol{G})=\frac{1}{\min _{k}\left(\left|1+\lambda_{\boldsymbol{K}}^{(k)}\right|\right)},
$$

with

$$
\left|1+\lambda_{\boldsymbol{K}}\right|=\sqrt{(1+2(1-\cos (\phi)) \sigma|\lambda|(1+\sigma|\lambda|))} \geq 1, \quad \forall \sigma .
$$

Denoting $v=\sigma|\lambda|$, we see that $v \longmapsto \rho(\boldsymbol{G})(\phi, v)$ is a strictly diminishing function and therefore

$$
\rho(\boldsymbol{G})=\frac{1}{\sqrt{\left(1+2(1-\cos (\phi)) v_{\min }\left(1+v_{\min }\right)\right)}},
$$

where $v_{\text {min }}=\sigma \min _{k}\left(\left|\lambda^{(k)}\right|\right)$ if no matrix time-stepping scheme is formulated, $v_{\min }=\min _{k}\left(\sigma^{(k)}\left|\lambda^{(k)}\right|\right)$, $\sigma^{(k)}=\frac{\Delta t^{(k)}}{\delta x}$ with the matrix time-stepping scheme. The optimal rate of convergence to a steady state is then obtained for large CFL numbers with $\left.\lim _{C F L \rightarrow \infty} \rho(\boldsymbol{G})=0, \forall \phi \in\right] 0,2 \pi[, \forall \lambda \neq 0$. The same results hold for the two-dimensional problem with the upper-bound estimate

$$
\rho(\boldsymbol{G}) \leq \frac{1}{\sqrt{\left(1+2(1-\cos (\phi))\left(v_{\min }\right)_{x}+2(1-\cos (\theta))\left(v_{\min }\right)_{y}\right.}} \leq 1, \quad \forall \sigma_{x}, \sigma_{y} .
$$

So the higher are $\left(v_{\min }\right)_{x}$ or $\left(v_{\min }\right)_{y}$, the lower is $\rho(\boldsymbol{G})$ and the better is the convergence rate to a steady state.

In the case of the rescaled Roe scheme, using similar arguments, we get in the one-dimensional case

$$
\rho(\boldsymbol{G})=\frac{1}{\sqrt{\left(1+(1-\cos (\phi))\left[2+(1-\cos (\phi))\left(v_{\mu}\right)_{\min }\right]\left(v_{\mu}\right)_{\min }+\sin ^{2}(\phi)\left(v_{\lambda}\right)_{\min }\right.}},
$$

where $\left(v_{\mu}\right)_{\min }=\sigma \min _{k}\left(\mu^{(k)}\right),\left(v_{\lambda}\right)_{\min }=\sigma \min _{k}\left(\left|\lambda^{(k)}\right|\right)$ if no matrix time-stepping scheme is formulated, $\left(v_{\mu}\right)_{\min }=\min _{k}\left(\sigma^{(k)} \mu^{(k)}\right),\left(v_{\lambda}\right)_{\min }=\min _{k}\left(\sigma^{(k)}\left|\lambda^{(k)}\right|\right)$ with the matrix time-stepping scheme.

Now comparing the effect of the matrix time-stepping scheme on the non-dimensional quantities $\left(v_{\min }\right)_{x}$ and $\left(v_{\min }\right)_{y}$ for both the Roe scheme and the rescaled Roe scheme, we get -For the Roe scheme:

$$
\sigma_{x}\left|\lambda_{\boldsymbol{A}}^{(k)}\right|=\frac{\Delta t}{\delta x}\left|\lambda_{\boldsymbol{A}}^{(k)}\right|=C F L \frac{h}{\delta x} \frac{\left|\lambda_{\boldsymbol{A}}^{(k)}\right|}{\rho(\boldsymbol{A})+\rho(\boldsymbol{B})} \leq C F L \frac{h}{\delta x} \frac{\left|\lambda_{\boldsymbol{A}}^{(k)}\right|}{\left|\lambda_{\boldsymbol{A}}^{(k)}\right|+\left|\lambda_{\boldsymbol{B}}^{(k)}\right|}=\frac{\Delta t^{(k)}}{\delta x}\left|\lambda_{\boldsymbol{A}}^{(k)}\right|=\sigma_{x}^{(k)}\left|\lambda_{\boldsymbol{A}}^{(k)}\right| \quad \forall k,
$$


and similarly $\sigma_{y}\left|\lambda_{\boldsymbol{B}}^{(k)}\right| \leq \sigma_{y}^{(k)}\left|\lambda_{\boldsymbol{B}}^{(k)}\right|, \forall k$. Assuming that $\left(v_{\min }\right)_{x}$ is reached for $k=k_{0}$, we see that

$$
\left(v_{\min }\right)_{x}=\sigma_{x}\left|\lambda_{\boldsymbol{A}}^{\left(k_{0}\right)}\right| \leq \sigma_{x}\left|\lambda_{\boldsymbol{A}}^{(k)}\right| \leq \sigma_{x}^{(k)}\left|\lambda_{\boldsymbol{A}}^{(k)}\right|, \quad \forall k \quad \Rightarrow \quad\left(v_{\min }\right)_{x} \leq \min _{k}\left(\sigma_{x}^{(k)}\left|\lambda_{\boldsymbol{A}}^{(k)}\right|\right) .
$$

-For the rescaled Roe scheme, the previous result writes

$$
\left[\left(v_{\mu}\right)_{\min }\right]_{x}=\sigma_{x} \mu_{\boldsymbol{A}}^{\left(k_{0}\right)} \leq \min _{k}\left(\sigma_{x}^{(k)} \mu_{\boldsymbol{A}}^{(k)}\right) \quad \text { and } \quad\left[\left(v_{\lambda}\right)_{\min }\right]_{x}=\sigma_{x}\left|\lambda_{\boldsymbol{A}}^{\left(k_{0}\right)}\right| \leq \min _{k}\left(\sigma_{x}^{(k)} \mid \lambda_{\boldsymbol{A}}^{(k)}\right) \mid .
$$

Therefore the matrix time-stepping scheme always yields higher $\left(v_{\min }\right)_{x}$ and $\left(v_{\min }\right)_{y}$ and thus may improve the damping properties of the iterative procedure. It is also interesting to look at the situation in the incompressible limit. Since for the Roe scheme in a fixed mesh

$$
\left|\lambda_{+}\right| \simeq\left|\lambda_{-}\right| \simeq O\left(\frac{1}{M}\right) \quad \text { and } \quad\left|\lambda_{0}\right| \simeq O(1) \quad \text { as } \quad M \rightarrow 0,
$$

with find that

$$
\left(v_{\min }\right)_{x} \simeq O(M)<\min _{k}\left(\sigma_{x}^{(k)}\left|\lambda^{(k)}\right|\right) \simeq O(1) \quad \text { as } \quad M \rightarrow 0 .
$$

For the rescaled Roe scheme in the same conditions

$$
\mu_{+} \simeq O\left(\frac{1}{M^{2}}\right) \quad \text { and } \quad \mu_{-} \simeq \mu_{0} \simeq O(1) \quad \text { as } \quad M \rightarrow 0,
$$

thus

$$
\left[\left(v_{\mu}\right)_{\min }\right]_{x} \simeq O\left(M^{2}\right)<<\min _{k}\left(\sigma_{x}^{(k)} \mu^{(k)}\right) \simeq O(1) \quad \text { as } \quad M \rightarrow 0 .
$$

So the optimal damping properties of the matrix time-stepping scheme should be enforced in the incompressible limit.

It can also be demonstrated that the rescaled Roe scheme is also second-order dissipative in the sens of Kreiss [25] and can be used for the computation of non-linear flows.

\subsection{Under relaxation of the implicit scheme}

In our implementation of the rescaled Roe scheme, we have considered the finite-volume method in structured grids. The implicit scheme (55) is solved iteratively using a LU-SGS method, with successive forward and backward diagonal sweeps within the mesh. Numerical experiments for low Mach-number flow simulations have shown that a large number of diagonal sweeps should be considered to get an efficient inversion of the linear system. Furthermore, considering that all boundary conditions were not linearized implicitly (only the implicit treatment of the wall and symmetry boundary conditions was implemented) and the error introduced the 
iterative procedure, this practically limits the use of large CFL numbers required by the stability condition. As shown in the previous section, the efficiency of rescaled Roe scheme to handle low-speed flow computations depends on first the use of large CFL numbers and second the use of a characteristic time-step matrix. This will be illustrated next for the case of the NACA0012 airfoil. Then, it has been found necessary to improve the efficiency the LU-SGS algorithm and a simple under-relaxation of the implicit scheme turned out to very efficient to overcome these limitations.

Let first reformulate the implicit scheme (55) in condensed form

$$
(I d-\Delta t M) \Delta W=\Delta t \operatorname{Res}^{e x p},
$$

where $\Delta \boldsymbol{t}=\Delta t \boldsymbol{I} \boldsymbol{d}$ if a scalar time step is used or $\boldsymbol{\Delta} \boldsymbol{t}=\boldsymbol{\Delta} \boldsymbol{t}_{\boldsymbol{c}}$ for the time-step matrix. We modify this with

$$
(I d-\phi \Delta t M) \Delta W=\Delta t \operatorname{Res}^{e x p},
$$

and we take $\phi>1$, which implies under-relaxation. So large values of $\phi$ will slow down the convergence rate for the steady problem, but will permit the use large CFL numbers. We experienced that, depending upon the low-Mach number inflow conditions, the relaxation coefficient $\phi$ should be increased from $\phi=1.5$ at $M_{\infty}=10^{-1}$ to $\phi=150$ at $M_{\infty}=10^{-3}$, in order to obtain a stable algorithm supporting very large CFL numbers, with typically $C F L \simeq O\left(1 / M_{\infty}^{2}\right)$.

\section{Practical implementation of the rescaled Roe scheme}

In our implementation, the Choi-Merkle preconditioning parameter will assume the following formulation

$$
\frac{\beta^{2}}{a^{2}}=\min \left(\max \left(M^{2}, \epsilon^{2}\right), 1\right)
$$

where $\epsilon^{2}$ is used as a small cut-off value. It is worth mentioning that for high subcritical or transonic flows, it is essential to set the small parameter $\epsilon^{2}$ to a much smaller value than $M_{\infty}^{2}$ to preserve the higher accuracy of the rescaled Roe scheme in the low-speed flow regions. For lowspeed flow computations, the small parameter $\epsilon^{2}$ was set to a cut-off value for all inflow Mach numbers considered. In [1], the use of the small parameter $\epsilon^{2}$ was not mentioned. This doesn't seem to create any numerical difficulties. On the other hand, no spurious dissipation effects due to corrected eigenvalues are introduced. For low Mach-number flow computations, the Van Leer 
formulation was also considered

$$
\frac{\beta^{2}}{a^{2}}=\min \left(\frac{2 M^{2}}{1-2 M^{2}}, 1\right), \quad \text { so } \quad \frac{\beta^{2}}{a^{2}}=1 \quad \text { as } \quad M \geq 0.5 .
$$

\subsection{Entropy fix}

The so-called entropy fix is considered as a mean to select the relevant physical solution satisfying the entropy condition to the hyperbolic problem, which may be violated by the Roe's approximate Riemann solver. The formulations proposed by Harten have been widely accepted by the CFD community, as being very simple to implement in existing CFD codes based on the Roe scheme. These formulations act on all the eigenvalues approaching zero and therefore also correct the contact discontinuity with consequently additional numerical dissipation and loss of accuracy. The behaviour of the modified eigenvalues $\mu_{ \pm}$and $\mu_{0}=|q|$ suggests to apply selectively the entropy fix. With $\mu_{+}>0$, a correction has to be found only for $\mu_{0}$ at the stagnation point and for $\mu_{-}$at the sonic line, since the formulation of the rescaled matrix prevents $\mu_{-}$from being too small near a stagnation point. With $\mu_{-} \simeq|q|$ near the stagnation point, this suggests to equalize $\mu_{0}$ and $\mu_{-}$when $M \rightarrow 0$. We suppose the Mach number to be small enough so that the preconditioning parameter recovers its cut-off value $\frac{\beta^{2}}{a^{2}}=\epsilon^{2}$. Then, a possible correction will assume the following form

$$
\mu_{0}=\max (|q|, \epsilon a|\boldsymbol{n}|) \quad \text { with } \quad \epsilon a|\boldsymbol{n}|=\lim _{M \rightarrow 0} \mu_{-} \simeq O(1)
$$

This correction might be interpreted as formally similar to the first entropy correction proposed by Harten and Hyman [26]. However, notice that the quantity $\epsilon a|\boldsymbol{n}|$ is actually a bit smaller than the threshold employed for the entropy fix, which is usually based on some fraction of the spectral radius $|q|+a|\boldsymbol{n}|$ (even for the linear waves) and not on the local speed of sound only. For viscous or turbulent flows, as it is discussed below, the formulation of the cut-off value $\epsilon^{2}$ must be reconsidered, and the correction (58) may assume a different formulation.

Regarding the acoustic eigenvalue $\mu_{-}$at the sonic line when $M_{n}=1$, it seems reasonable to be consistent with the formulation of the original matrix dissipation, by considering the same formulation of the entropy fix as used for the Roe scheme. This choice is motivated by the extension to a supersonic flow described in the following. Considering again the symmetrizing 
entropic variables, in the case of subsonic flow $|u| \leq a$ we have the following diagonalization

$$
\left|\tilde{\boldsymbol{A}}_{\mathbf{0}}\right|=\left(\begin{array}{cc}
a & a \\
a & -a
\end{array}\right)\left(\begin{array}{cc}
|u+a| & 0 \\
0 & |u-a|
\end{array}\right)\left(\begin{array}{cc}
\frac{1}{2 a} & \frac{1}{2 a} \\
\frac{1}{2 a} & -\frac{1}{2 a}
\end{array}\right) .
$$

Although in case of transonic flow the entropic variables are not appropriate, suppose for the analysis that $u \rightarrow a$. Then the acoustic eigenvalue $\left|\lambda_{-}\right|=|u-a|$ may be corrected by some entropy fix and therefore $\left|\lambda_{-}\right| \rightarrow 0$ is modified with $\left|\tilde{A}_{\mathbf{0}}\right|$ formulated for $\operatorname{diag}(|u+a|, \Psi(|u-a|))$, where $\Psi$ is some given cut-off function preventing $\left|\lambda_{-}\right|$from reaching zero. On the other hand, assuming $\frac{\beta^{2}}{a^{2}} \rightarrow 1$ with $\alpha=0$ in the expression of the rescaled dissipation matrix as $|u| \rightarrow a$, we get

$$
\tilde{\boldsymbol{P}}_{\mathbf{0}}^{-1}\left|\tilde{\boldsymbol{P}}_{\mathbf{0}} \tilde{\boldsymbol{A}}_{\mathbf{0}}\right|=\left(\begin{array}{cc}
|u| & u \\
u & -|u|
\end{array}\right)\left(\begin{array}{cc}
a+|u| & 0 \\
0 & a-|u|
\end{array}\right)\left(\begin{array}{cc}
\frac{1}{2|u|} & \frac{1}{2 u} \\
\frac{1}{2 u} & -\frac{1}{2|u|}
\end{array}\right) .
$$

Thus at the sonic line, $\mu_{-}=a-|u| \rightarrow 0$ and $\tilde{\boldsymbol{P}}_{\mathbf{0}}{ }^{-1}\left|\tilde{\boldsymbol{P}}_{\mathbf{0}} \tilde{\boldsymbol{A}}_{\mathbf{0}}\right|$ may be also reformulated with $\operatorname{diag}(a+$ $\left.|u|, \Psi_{p}(a-|u|)\right)$. The cut-off function $\Psi_{p}\left(\mu_{-}\right)$must be defined in order to assure $\tilde{\boldsymbol{P}}_{\mathbf{0}}^{-1}\left|\tilde{\boldsymbol{P}}_{\mathbf{0}} \tilde{\boldsymbol{A}}_{\mathbf{0}}\right| \rightarrow\left|\tilde{\boldsymbol{A}}_{\mathbf{0}}\right|$ when $|u| \rightarrow a$. In the limit $|u| \rightarrow a$, when applying the entropy fix, we can see that we get the identity $\tilde{\boldsymbol{P}}_{\mathbf{0}}^{-1}\left|\tilde{\boldsymbol{P}}_{\mathbf{0}} \tilde{\boldsymbol{A}}_{\mathbf{0}}\right|=\left|\tilde{\boldsymbol{A}}_{\mathbf{0}}\right|$ provided that

$$
\left\{\begin{array}{l}
\Psi_{p}\left(\mu_{-}\right)=\Psi\left(\left|\lambda_{+}\right|\right) \text {and } \mu_{+}=\left|\lambda_{-}\right| \text {if } u \rightarrow-a \\
\Psi_{p}\left(\mu_{-}\right)=\Psi\left(\left|\lambda_{-}\right|\right) \text {and } \mu_{+}=\left|\lambda_{+}\right| \text {if } u \rightarrow a
\end{array}\right.
$$

If no entropy fix is applied

$$
\left|\tilde{\boldsymbol{A}}_{\mathbf{0}}\right|=\tilde{\boldsymbol{P}}_{\mathbf{0}}^{-1}\left|\tilde{\boldsymbol{P}}_{\mathbf{0}} \tilde{\boldsymbol{A}}_{\mathbf{0}}\right|=\left(\begin{array}{cc}
a & \pm a \\
& \\
\pm a & a
\end{array}\right) \text { when } u= \pm a .
$$

The formulation (59) of the entropy fix for the rescaled Roe scheme is unchanged by similarity transformations and can be also extended to the Euler equations in conservation form. The definition of $\Psi_{p}$ is also formally unchanged in the multidimensional case, as indicated below. For complex non-linear flows, we may consider the classical Harten's entropy fix formulated in [27]

$$
\left\{\begin{array}{llll}
\Psi(|\lambda|)= & \frac{\lambda^{2}+\delta_{h}^{2}}{2 \delta_{h}} & \text { if } & |\lambda|<\delta_{h} \\
\Psi(|\lambda|)= & |\lambda| & \text { if } & |\lambda| \geq \delta_{h}
\end{array}\right.
$$


with $\delta_{h}$ being the threshold for the correction of the eigenvalues approaching zero which is usually a fraction of $\rho(\boldsymbol{A})$.

\subsection{Supersonic flow}

If the flow becomes locally supersonic, the formulation of the rescaled Roe scheme is no longer valid and the original Roe scheme must be selected. It becomes then necessary to introduce a "switch" between the subsonic and supersonic flow conditions. The matrix valued dissipation $\boldsymbol{Q}$ must be formulated as follows

$$
\text { if } a|\boldsymbol{n}|-|q| \geq 0 \text { then } \boldsymbol{Q}=\boldsymbol{P}^{-\mathbf{1}}|\boldsymbol{P} \boldsymbol{A}| \text { else } \boldsymbol{Q}=\left|\boldsymbol{A}_{\text {roe }}\right| \text {, }
$$

with $\boldsymbol{P}^{-\mathbf{1}}|\boldsymbol{P A}|=\left|\boldsymbol{A}_{\text {roe }}\right|$ if $|q|=a|\boldsymbol{n}|$ and $\alpha=0$ provided that the rescaled dissipation matrix is formulated for the Roe's average. In the supersonic flow regions, the CFL condition must be selected accordingly with (53). As aforementioned, the entropy fix must be applied selectively in order to assure a smooth transition with the Roe scheme near the sonic line. For the rescaled dissipation matrix, the entropy fix must be applied according to (59) with

$$
\Psi_{p}\left(\mu_{-}\right)=\Psi\left(\left|\lambda_{ \pm}\right|\right) \quad \text { and } \quad \mu_{+}=\left|\lambda_{\mp}\right| \quad \text { if } \quad|q \pm a| \boldsymbol{n}|| \leq \delta_{h},
$$

whenever the Harten's entropy fix (60) is considered.

Since $\mu_{+} \rightarrow \rho(\boldsymbol{A})$ as $M \rightarrow 1$, no special treatment has to be considered for the formulation of the scalar time-step. However, this is not true for the eigenvalues as illustrated in Fig. 1-3. So in the formulation of the time-step matrix, a smooth transition $\boldsymbol{A}_{p}=\boldsymbol{R}_{A} \tilde{\boldsymbol{\Lambda}}_{A} \boldsymbol{R}_{A}{ }^{-1} \rightarrow\left|\boldsymbol{A}_{\text {roe }}\right|$ can only be achieved by modifying the eigenvalues $\mu^{(k)}$. A possible reformulation of the acoustic speeds $\mu_{ \pm}^{*}$ has been found by considering the "acoustic portion" of decomposition (43). Upon substitution of matrices $\boldsymbol{A}$ and $\boldsymbol{P}^{-1}$ by their respective eigenvalues, we get

$$
\mu_{+}^{*}=Q_{11}+a|\boldsymbol{n}| \frac{z q}{\sqrt{\chi}}, \quad \mu_{-}^{*}=\left|Q_{22}-a\right| \boldsymbol{n}\left|\frac{z q}{\sqrt{\chi}}\right|,
$$

where the absolute value on $\mu_{-}^{*}$ ensures positivity. The behaviour of the corrected eigenvalues as function of $\frac{q}{a|\boldsymbol{n}|}$ is plotted in Fig. 4 for $M=0.1$ and $\alpha=0$. It can be especially seen that $\mu_{ \pm}^{*} \rightarrow\left|\lambda_{ \pm}\right|$the eigenvalues of $\left|\boldsymbol{A}_{\text {roe }}\right|$ as $q \rightarrow \pm a|\boldsymbol{n}|$ and that the asymptotic behaviour of $\mu_{+}^{*} \simeq \mu_{+}$ as $|q| \rightarrow 0$ is reproduced by the correction. Note that $\mu_{-}^{*} \simeq\left|\lambda_{-}\right|$except when $|q| \rightarrow 0$ where $\mu_{-}^{*}$ becomes singular. So the final expression of $\mu_{-}^{*}$ is further simplified in order to avoid any singularity, and we simply take $\mu_{-}^{*}=\left|\lambda_{-}\right|$in the whole subsonic range $|q| \leq a|\boldsymbol{n}|$. So for the 
rescaled Roe scheme, the time-step matrix will assume the following expression for all inflow Mach numbers

$$
\Delta t^{+}=C F L \frac{h}{Q_{11}+a|\boldsymbol{n}| \frac{z q}{\sqrt{\chi}}}, \quad \Delta t^{-}=C F L \frac{h}{|q-a| \boldsymbol{n}||}, \quad \Delta t^{0}=C F L \frac{h}{|q|} .
$$

In practice, $|q-a| \boldsymbol{n} \|$ and $|q|$ may locally vanish or be very small and must be bounded away from zero with the entropy fix.

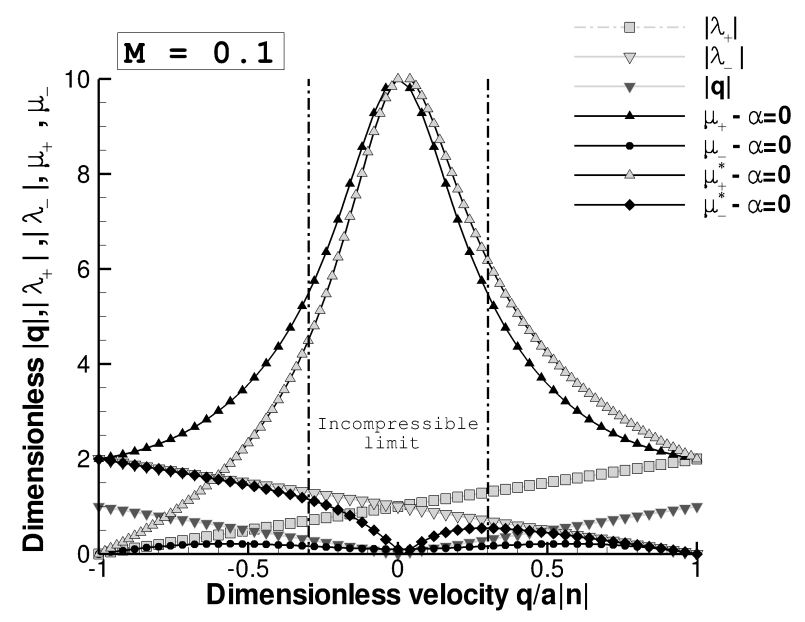

Figure 4: Dimensionless corrected eigenvalues $\mu_{ \pm}^{*}$ in the definition of time-step matrix when $M=0.1$ - Grey delta symbols: $\mu_{+}^{*}$. Black diamond symbols: $\mu_{-}^{*}$

\subsection{High-order extension}

The rescaled Roe scheme can be easily extended to higher orders of space accuracy assuring the TVD property by using the MUSCL extrapolation, since it doesn't affect the formulation of the matrix valued dissipation.

\subsection{Extension to viscous flows}

The local preconditioning approach used to construct the rescaled dissipation matrix (whatever it is iterative or not) may be further developed to handle viscous and turbulent flows. So far, the extension to the Navier-Stokes/RANS equations has been considered by extending the definition of the preconditioning parameter $\frac{\beta^{2}}{a^{2}}$ in order to enforce the accuracy and especially the robustness of the preconditioned scheme in stagnation point regions and in the boundary layer 
with high-aspect ratio grids. It has been especially demonstrated in [28] that a better control of the free parameter $\frac{\beta^{2}}{a^{2}}$ has to be achieved in the low Reynolds number flow regions. The authors suggest the cut-off value $\epsilon^{2}$ to be related to the isentropic Mach number inside the boundary layer. In [29], the "inviscid" preconditioning parameter is modified for high lift configurations and a cell Reynolds number is considered as a viscous correction. The necessary corrections of the original formulation are especially relevant for the computation of some industrial configurations characterized by co-existing transonic flows and large three-dimensional separated flows. These modifications obviously do not modify the derivation of the eigenspaces and the formulation of stability condition for the rescaled dissipation matrix, the low-speed preconditioner remaining formally the same.

\section{Computational results}

We were basically interested in testing numerically the stability and the accuracy of the rescaled matrix dissipation for the Roe scheme in the two extreme cases $M \rightarrow 0$ and $M \rightarrow 1$. Since it was necessary to assess the effect of removing the iterative preconditioning on both the numerical stability and the accuracy of the solution, flow configurations were selected assuming steady conditions. In order to frame the assumption of inviscid flow considered previously and to demonstrate numerically the properties of the rescaled Roe scheme, we have considered a NACA0012 airfoil configuration widely used for the assessment of CFD solvers. The meshes used are the Vasseberg-Jameson meshes, a family of O-meshes generated from a conformal transformation, yielding quadrilateral cells with an aspect ratio of one, with gridlines essentially orthogonal at each vertex within the mesh [30]. This mesh topology allows to avoid introducing poor grid quality or highly grid stretching effects in the numerical solutions. Note that the original formulation in the CFD solver of the boundary conditions using characteristic variables has been left unchanged when the rescaled Roe scheme was selected.

\subsection{Low Mach number flows about the NACA0012 airfoil}

The experiment of reference [1] was reproduced for the reformulation of the rescaled Roe matrix dissipation. In [1] the rescaled dissipation matrix was computed from the diagonalization of preconditioned Jacobian matrix $|\boldsymbol{P A}|$, while in our implementation, the diagonalization was formulated from the full matrix $\boldsymbol{P}^{-1}|\boldsymbol{P A}|$ and the implicit scheme (55) with matrix coefficients 
(56) was used. The primary interest of this test case is to demonstrate that the proper scaling of the pressure field is recovered at the discrete level in the incompressible limit by the rescaled Roe scheme. The test-case corresponds to a nonlifting solution at zero angle-of-attack. The grid considered for this preliminary assessment has $N_{c}=128$ cells in both directions.

For the normalized equations, we know that the discrete solutions of the first-order Roe scheme support pressure fluctuations in space of order $M_{\infty}$, with $p(\boldsymbol{x}, t)=p_{0}(t)+p_{1}(\boldsymbol{x}, t) M_{\infty}$, while in the continuous case, the pressure fluctuations scale as $M_{\infty}^{2}$. It was also demonstrated that the rescaled Roe scheme (with $\alpha=0$ ) support pressure fluctuations in space of secondorder $p(\boldsymbol{x}, t)=p_{0}(t)+p_{2}(\boldsymbol{x}, t) M_{\infty}^{2}$. Thus, without preconditioning or rescaling of the dissipation operator, a compressible flow solver usually fails to compute low Mach number flows.

A rigorous way to prove this result numerically consists in considering a sequence of computations with decreasing inflow Mach number using the same mesh and investigating whether the pressure fluctuations undergo a linear or quadratic behaviour with the Mach number. Looking for steady solutions, the pressure field will then assume the form $p(\boldsymbol{x})=p_{0}+\delta p(\boldsymbol{x})$, with $\delta p(\boldsymbol{x})=p_{f}(\boldsymbol{x}) M_{\infty}^{n}$, where $p_{0}$ is the leading order pressure corresponding to the thermodynamic static pressure, $p_{f}$ is the magnitude of the pressure fluctuations and $n=1$ or 2 is the order of the fluctuations. Following [1], the accuracy of the computations in the incompressible limit can be characterized by the evolution of a normalized pressure field $\tilde{p} \in[0,1]$ and an indicator characterizing the order of the pressure fluctuations as the Mach number goes to zero. The normalized pressure used for the comparison of the pressure fields at different inflow Mach numbers is defined as

$$
\tilde{p}(\boldsymbol{x})=\frac{p(\boldsymbol{x})-p_{\min }}{p_{\max }-p_{\min }}=\frac{p_{f}(\boldsymbol{x})-\left(p_{f}\right)_{\max }}{\left(p_{f}\right)_{\max }-\left(p_{f}\right)_{\min }}=\tilde{p}_{f}(\boldsymbol{x}),
$$

and then also corresponds to a normalized field for pressure fluctuations. Note that the normalized pressure $\tilde{p}$ is independent from the inflow Mach number and therefore as $M_{\infty} \rightarrow 0$, numerical solutions should also converge to a consistent approximation of the incompressible solution. A normalized pressure fluctuation $\delta \tilde{p}$ is also introduced with

$$
\delta \tilde{p}=\frac{p_{\max }-p_{\min }}{p_{\max }} \simeq\left[\frac{\left(p_{f}\right)_{\max }-\left(p_{f}\right)_{\min }}{p_{0}}\right] M_{\infty}^{n} \quad \text { for } \quad M_{\infty}<<1 .
$$

Since in the normalization process all flow variables are of the same order of magnitude (around unity), the above definition of $\delta \tilde{p}$ gives the variation of the pressure fluctuations with the Mach number. 
The behaviour $p=O\left(1 / M^{2}\right)$ for the pressure field was reproduced for a fixed mesh within a sequence of computations by increasing the inflow pressure while keeping constant the inflow velocity and the density, for decreasing Mach numbers $M_{\infty}$ in the range $10^{-1}$ to $10^{-3}$. The entropy correction was deactivated for all inflow Mach numbers considered. Numerical experiments are represented in the next figures for the Van Leer formulation of the preconditioning parameter and $\alpha=-1$. As expected, solutions obtained with the rescaled matrix dissipation converge to a unique isentropic solution with constant density, corresponding to a decoupling of the velocity and pressure fields, which cannot be achieved with the Roe scheme, as illustrated in Fig. 5-6. The behaviour of the pressure fluctuations with the inflow Mach number is represented in figure 7. With the rescaled Roe scheme, the pressure fluctuations exactly scale with $M_{\infty}^{2}$. As also indicated, the same experience was conducted for the Choi-Merkle formulation of the preconditioning parameter and $\alpha=0$, with exactly the same quadratic behaviour reproduced for the pressure fluctuations. As shown by many authors, we see that the Roe scheme supports fluctuations of order $M_{\infty}$ in the incompressible limit. Additionally, for inflow Mach numbers lower than 0.1 , a grid-convergence study carried out in [2] rigorously demonstrates that the effort in grid density required to the Roe scheme to consistently converge to the correct pressure field is inversely proportional to the Mach number. This is a consequence of the behaviour of the leading order term of the truncation error in the incompressible limit.

The effects of the CFL number and of the use of the matrix time-stepping scheme are illustrated in Fig 8 for the rescaled Roe scheme. As demonstrated in the stability analysis, the convergence rate is drastically increased when large CFL numbers are used, and the time-step matrix proved to be more efficient than the standard scalar time for any tested CFL number. The use of large CFL numbers was made possible by under relaxation of the implicit scheme. Largest CFL numbers correspond to $\Delta t_{+} \simeq O(h)$, so typically $\mathrm{CFL} \simeq O\left(1 / M_{\infty}^{2}\right)$, with $\mathrm{CFL}=100$ $(\phi=1)$ or larger for $M_{\infty}=10^{-1}, \mathrm{CFL}=10000(\phi=15)$ for $M_{\infty}=10^{-2}$ and CFL $=1000$ $000(\phi=150)$ for $M_{\infty}=10^{-3}$. In addition, as indicated in the figures with the dashed line, the zero level machine is reached for the pressure fluctuations of order $O\left(M^{-2}\right)$. Therefore, the zero level machine corresponding to the limit cycle is shifted according to the inflow Mach number, from 13 orders of convergence when $M_{\infty}=10^{-1}$ for pressure fluctuations of order $O\left(10^{-2}\right)$, to 9 orders of convergence when $M_{\infty}=10^{-3}$, with pressure fluctuations of order $O\left(10^{-6}\right)$.

The effect of a usual grid stretching was also investigated for the rescaled Roe scheme. The 


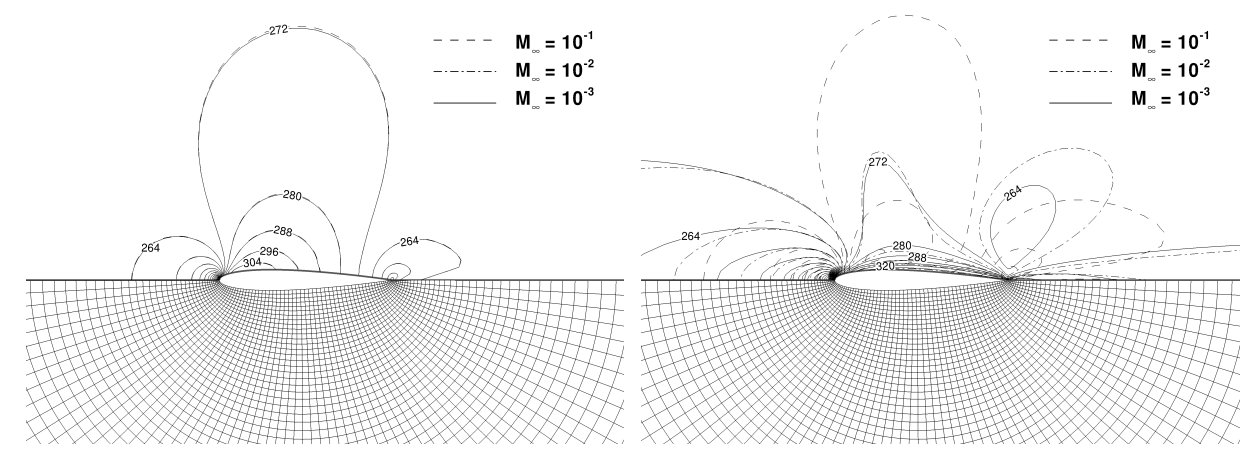

Figure 5: Velocity contours with the inflow Mach number in the Vasseberg-Jameson mesh. Left: rescaled Roe scheme. Right: Roe scheme $\left(V_{\infty}=270 \mathrm{~m} / \mathrm{s}\right)$

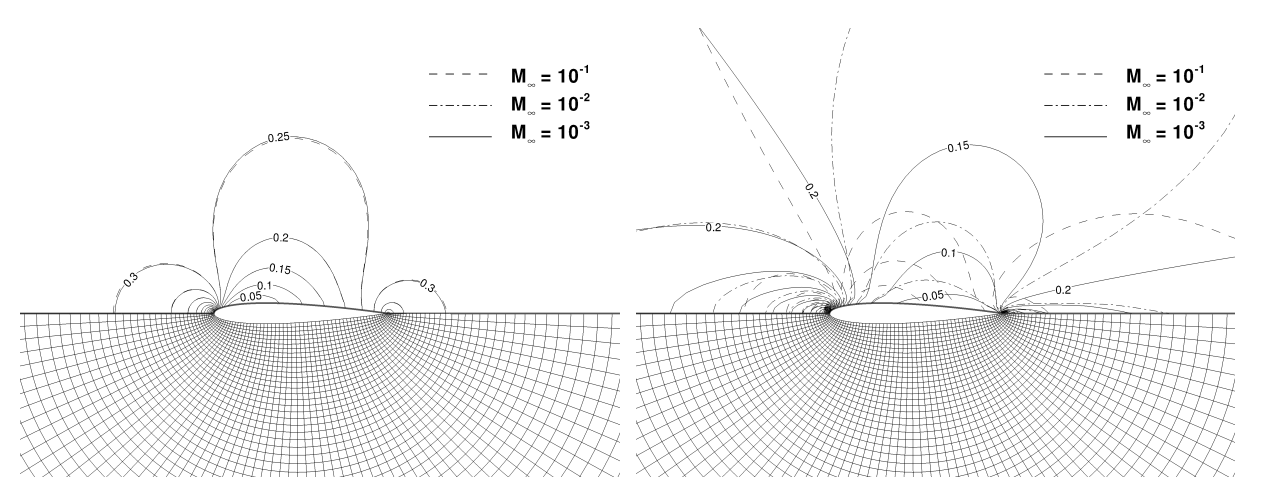

Figure 6: Contours of the normalized pressure with the inflow Mach number in the Vasseberg-Jameson mesh. Left: rescaled Roe scheme. Right: Roe scheme

unique isentropic solution for the velocity field and the convergence history are plotted in Fig. 9. Much lower CFL values could be used in this conventional grid, especially for $M_{\infty}=10^{-3}$. However, the effect of the time-step matrix on the convergence rate turned out to be much more important. The convergence rate is more than 30 times faster with the characteristic time-step matrix for $M_{\infty}=10^{-1}$, and practically, the zero machine level could not be reached with a reasonable number of time steps using the scalar time step, when $M_{\infty} \leq 10^{-2}$.

\subsection{Transonic flow about the NACA0012 airfoil}

A transonic flow condition for $M_{\infty}=0.8, \alpha=0^{0}$ was also considered in order to assess the smooth transition of the rescaled Roe scheme with the original Roe scheme at the sonic line, and the effect of the rescaled matrix dissipation at the stagnation point. This was achieved 


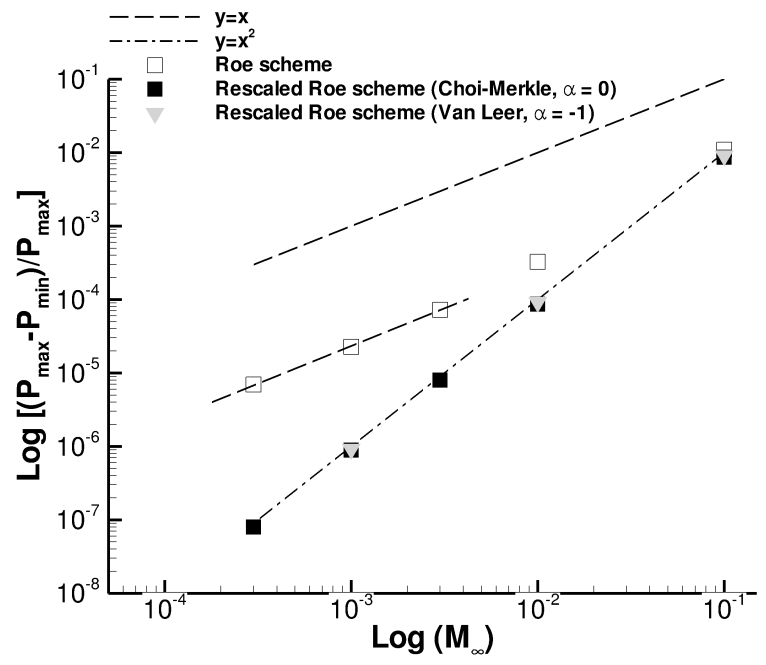

Figure 7: Pressure fluctuations with the inflow Mach number for the Roe scheme and the rescaled Roe scheme

within grid convergence, considering 5 out of the 8 coarsest-to-finest meshes defined in [30], with dimensions $128 \times 128$ to $2048 \times 2048$ cells. So the finest grid used is composed of over 4 million cells. For the rescaled Roe scheme, the Choi-Merkle formulation of the preconditioning parameter was selected, with $\epsilon^{2}=0.01<<M_{\infty}^{2}$ and $\alpha=0$. For both schemes, the entropy fix was activated, with the special treatment described previously for the rescaled Roe scheme, and a second-order MUSCL extrapolation was considered. For a transonic flow, both schemes have a similar conditioning and therefore similar convergence rates were obtained, with zero-level machine convergence obtained for each mesh. Note that with the matrix time-stepping scheme, the Roe scheme also achieves a faster convergence to the steady state compared to the standard scalar time step.

The comparison for the pressure coefficient obtained in the finest mesh is plotted in Fig. 10left. The respective solutions have a very similar discrete shock structure, with the same computed shock location. The effect of the proper scaling of the matrix dissipation has to be found near the stagnation point, where a slightly higher peak pressure is predicted with the rescaled Roe scheme. Then, the suction effect on the leading edge is slightly reduced and a larger pressure drag coefficient is found with the rescaled Roe scheme, for all meshes used in the grid-convergence study. A consequence of a better control of the numerical dissipation can be readily pointed out with the entropy distribution on the airfoil. The effect of the grid refinement on the entropy 

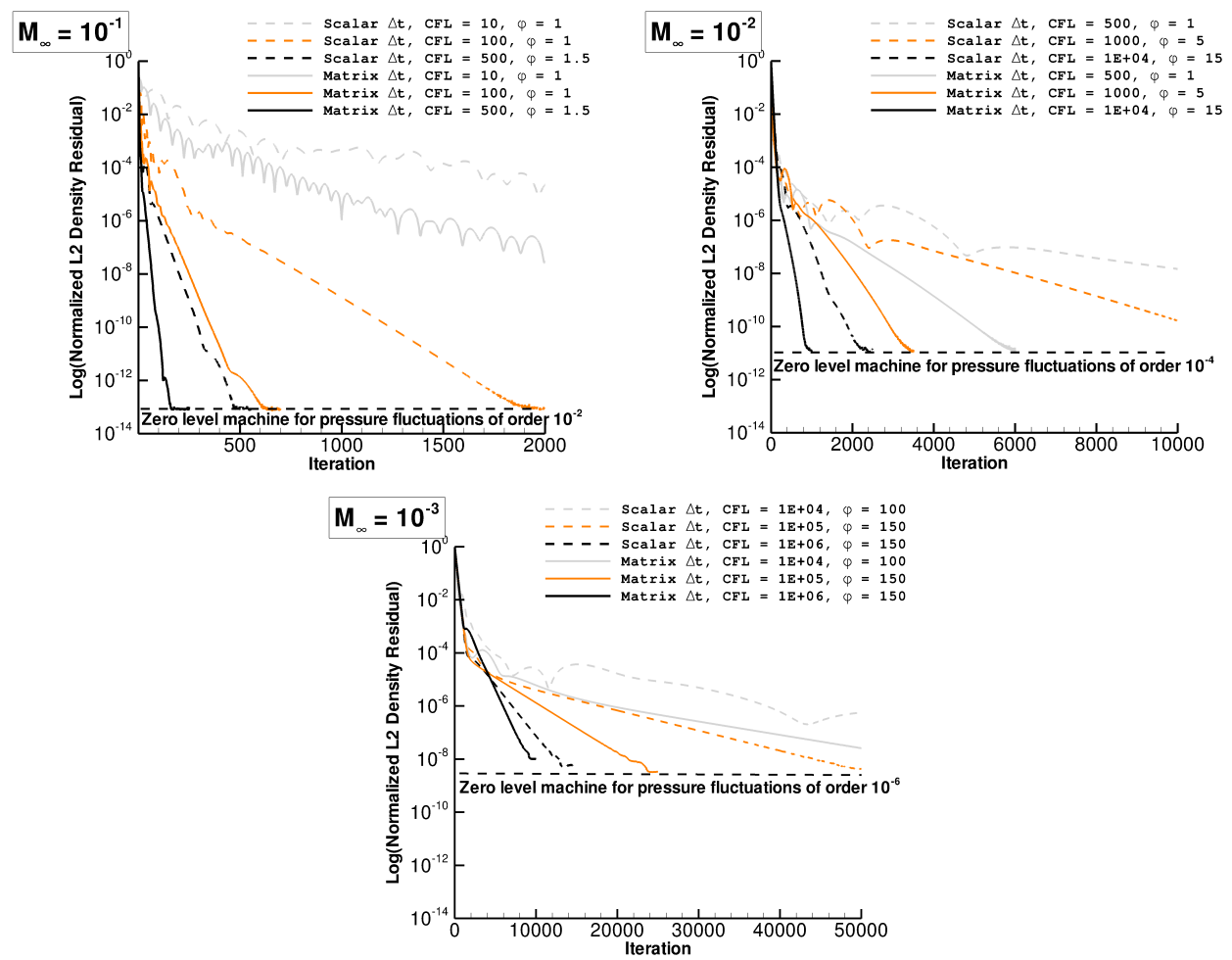

Figure 8: Convergence history for the rescaled Roe scheme with the inflow Mach number $10^{-1}, 10^{-2}$ and $10^{-3}$. Solid lines: matrix time-step. Dashed lines: scalar time step
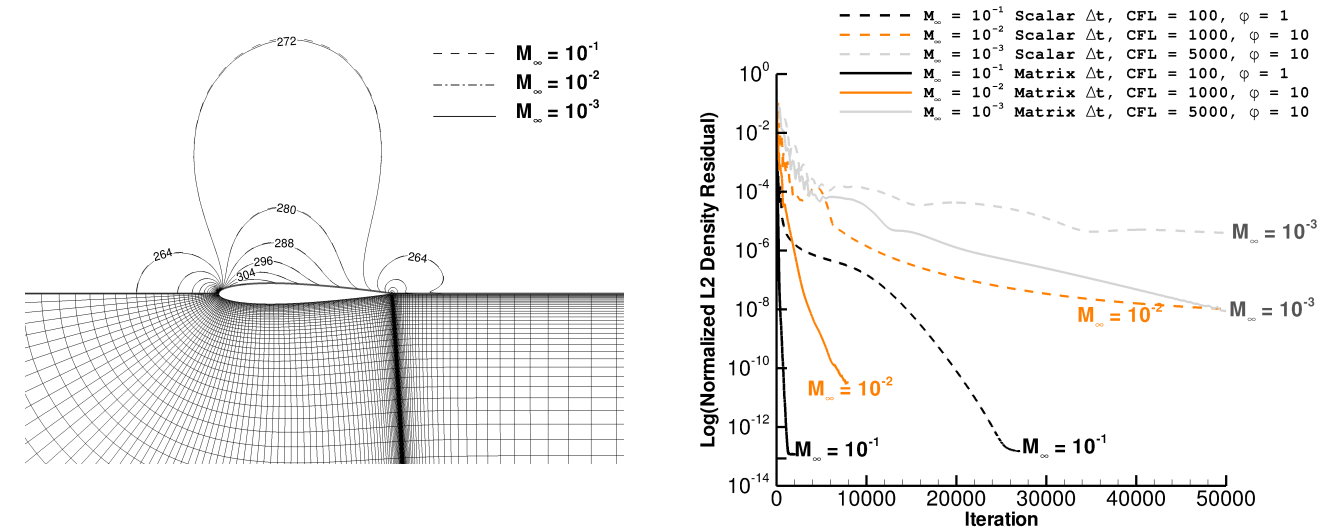

Figure 9: Effect of grid stretching on the rescaled Roe scheme. Left: Velocity contours with the inflow Mach number. Right: Convergence history for the scalar (dashed lines) and the matrix (solid lines) time steps 

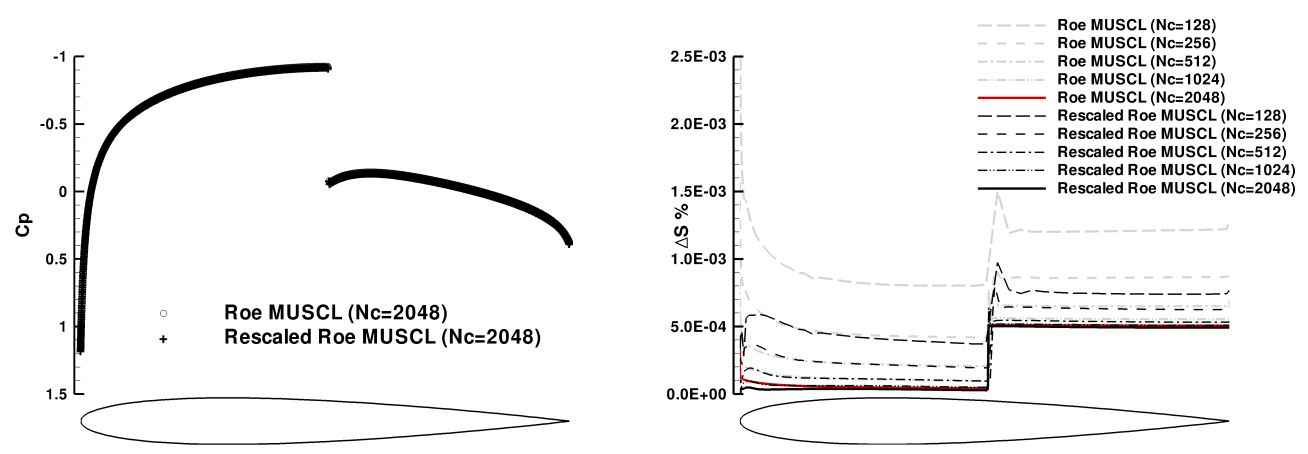

Figure 10: Comparison of the flow solution for the Roe scheme and the rescaled Roe scheme at $M_{\infty}=0.8, \alpha=0^{0}$. Left: pressure coefficient. Right: entropy distribution

distribution is illustrated in Fig. 10-right for both schemes. A significant effect of the rescaled matrix dissipation can be especially observed on the spurious entropy generation at the stagnation point. Since for both schemes, the same definition of the entropy fix was considered, the strong reduction of the spurious entropy is the consequence of the rescaling of the Roe scheme in the low speed flow regions where high gradients of the solution are present. Similar effects were observed in sub-critical and in low-speed flow conditions.

\section{Conclusions}

A rescaled matrix-valued dissipation was formulated by deriving explicitly the eigenspaces of the Roe-Turkel matrix dissipation. This formulation is interpreted as a rescaling of the Roe scheme for low Mach number flows. When the entropic variables are considered as independent variables, it is demonstrated that the rescaled matrix dissipation is positive semidefinite and can be diagonalized by unitary congruence. So, robustness problems could be significantly alleviated especially at stagnation points. However, our final objective is to developed a more accurate compressible flow solver in low speed flow regions, and the optimal normality properties of the rescaled matrix dissipation are lost by any change of variables. By construction, the rescaled Roe scheme gives the correct asymptotic order for the artificial dissipation terms, and in particular returns the proper quadratic behaviour of the pressure fluctuations in the incompressible limit. However, as the Mach number goes to zero, the system becomes very stiff and very small time 
steps must be used, independently of the definition of the local preconditioner used for the formulation of the Roe-Turkel scheme. With the rescaling of the Roe scheme, it is then necessary to completely reformulate the stability condition. A CFL condition was formulated explicitly, based on the spectral radius of the rescaled matrix dissipation. For steady-state problems, it is shown that a robust implicit scheme can be formulated in order to circumvent the severe stability bound occurring for low Mach number flows. The implicit scheme is characterized by the introduction of a characteristic time-step matrix, enforcing the damping properties of the scheme, and under-relaxation, allowing the use of very large CFL numbers. However, as indicated in the introduction, the rescaled Roe scheme is intended to improve locally the prediction of complex high Reynolds number flows characterized by the coexistence of high gradients of the solution in mixed incompressible and compressible regions, arising typically in industrial configurations. For such complex flows, it is anticipated that the overall stability of the numerical procedure will not be dominated by the limited number of computational cells where the flowfield can be retained as incompressible. In transonic flow conditions, the rescaled Roe scheme ensures a smooth transition with the original Roe scheme, provided that the entropy fix is carefully formulated. A grid-convergence study demonstrates that the discrete shock structure is preserved with the TVD property enforced by the MUSCL extrapolation. As a consequence of the proper scaling of the matrix dissipation, a significant effect can be especially observed for the inviscid flow, with a drastic reduction of the spurious entropy generation at the stagnation point.

\section{Acknowledgements}

The author is grateful to his colleagues Lionel Castillon, Christelle Wervaecke and Antoine Dumont for providing the 2D CFD code together with many useful test-cases. The author would also like to thank Daniel Destarac for providing the Vasseberg-Jameson conformal grids.

\section{References}

[1] H. Guillard, C. Viozat On The Behaviour of Upwind Schemes in The Low Mach Number Limit Computers \& Fluids, 28 (1999), 63-86

[2] C. Viozat Implicit Upwind Schemes for Low Mach Number Compressible Flows INRIA Report No 3084, January 1997

[3] X.S. Li, C.W. Gu An All-Speed Roe-Type Scheme and its Asymptotic Analysis of Low Mach Number Behaviour J. Comput. Phys., 227 (2008), 5144-5159 
[4] F. Rieper A Low Mach Number Fix for Roe's Approximate Riemann Solver J. Comput. Phys., 230 (2011), 52635287

[5] I. Mary, P.Sagaut, M. Deville An Algorithm for Unsteady Viscous Flows at all Speeds Int. J. Numer. Meth. Fluids, Vol. 34 (2000), pp. 371401

[6] H. Guillard, A. Murone On the Behaviour of Upwind Schemes in the Low Mach Number Limit: II. Godunov Type Schemes INRIA report, No 4189, May 2001

[7] X.S. Li, C.W. Gu Mechanism of Roe-type Schemes for All-Speed Flows and its Application Computers \& Fluids, 86 (2013), 56-70

[8] S. Dellacherie Analysis of Godunov Type Schemes Applied to The Compressible Euler System at Low Mach Number J. Comput. Phys., 229 (2010), 978-1016

[9] P. Birken, A. Meister Stability of Preconditioned Finite-Volume Schemes at Low Mach Number BIT Numerical Mathematics, 45, (2005), 463-480

[10] B. Thornber, A. Mosedale, D. Drikakis, D. Youngs, and R. Williams An Improved Reconstruction Method for Compressible Flows with Low Mach Number Features J. Comput. Phys., 227 (2008), 4873-4894

[11] K. Oßwald, A. Siegmund, P. Birken, V. Hannemann and A. Meister L2 Roe: a Low Dissipation Version of Roe's Approximate Riemann Solver for Low Mach Numbers Int. J. Numer. Meth. Fluids, (2015), DOI: 10.1002/fld.4175

[12] E. Turkel Review of Preconditioning Methods For Fluid Dynamics NASA CR 189712 (1992)

[13] E. Turkel, R. Radespiel and N. Kroll Assessment of Preconditioning Methods For Multidimensional Aerodynamics Computers \& Fluids, 6 (1997), 613-634

[14] Turkel E. Preconditioned Methods For Solving the Incompressible And Low Speed Compressible Equations J. Comput. Phys., 72 (1987), 277-298

[15] E. Turkel, V.N. Vatsa, R.Radespiel Preconditioning Methods For Low Speed Flows NASA Contractor Report 201605 and ICASE Report 96-57, October 1996

[16] E. Turkel, V.N. Vatsa Choice of Variables and Preconditioning for Time Dependent Problems AIAA paper $2003-$ 3692, 2003

[17] Turkel E. Preconditioned Techniques in Computational Fluid Dynamics Annual Review of Fluid Mechanics (1999) Vol. 31, pp. 385-416

[18] E. Turkel, A. Fiterman, and B. Van Leer Preconditioning and the limit of the compressible to the incompressible flow equations for finite difference schemes eds. M.M. Hafez and D.A. Caughey (Wiley, Chichester, 1994), pp. 215-234

[19] H. Guillard - A. Murrone On the Behaviour of Upwind Schemes in the Low Mach Number Limit : II. Godunov Type Schemes INRIA Report No 4189, May 2001

[20] Choi Y.H. Merkle C.L. The Application of Preconditioning To Viscous Flows J. Comput. Phys., 105 (1993), $207-$ 223

[21] D.L. Darmofal and J. Schmid The Importance of Eigenvectors for Local Preconditioners of the Euler Equations J. Comput. Phys., 127 (1996), 346-362

[22] B. Van Leer, W.T. Lee and P. Roe Characteristic Time-Stepping or Local Preconditioning of the Euler Equations AIAA-91-1552-CP (1991)

[23] W. A. Mulder and B. Van Leer Experiments with Implicit Upwind Methods for the Euler Equations J. Comput. 
Phys., 59 (1985), 232-246

[24] Lerat, A. Multidimensional Centered Schemes of the Lax-Wendroff Type Computational Fluid Dynamics Review, pp. 124-140, 1995

[25] A. Lerat Difference Schemes for Nonlinear Hyperbolic Systems - A General Framework Lecture Notes in Math., 1402, pp. 12-29, 1989

[26] A. Harten and J.M. Hyman Self Adjusting Grid Methods for one-dimensional hyperbolic conservation laws J. Comput. Phys., 50 (1983), 253-269

[27] A. Harten High Resolution Schemes for Hyperbolic Conservation Laws J. Comput. Phys., 49 (1983), 357-393

[28] Y. Colin, H. Deniau and J.F. Boussuge. A Robust Low-Speed Preconditioning Formulation Computers \& Fluids, 47 (2011), 1-15

[29] E. Turkel Robust Low Speed Preconditioning for Viscous High Lift Flows AIAA paper 2002-0962, 2002

[30] J. Vassberg and A. Jameson In Pursuit of Grid Convergence for Two-Dimensional Euler Solutions Journal of Aircraf, Vol. 47, No. 4, 2010 